\title{
Fitting LINER nuclei within the AGN family: A matter of obscuration?
}

\author{
O. González-Martín ${ }^{1}$ \\ Physics Department, University of Crete, PO Box 2208, 71003 Heraklion, Crete, Greece \\ X-ray Astronomy Group, Department of Physics and Astronomy, Leicester University, \\ Leicester LE1 7RH, UK \\ omaira@physics.uoc.gr \\ J. Masegosa and I. Márquez \\ Instituto de Astrofísica de Andalucía (CSIC), Granada, SPAIN \\ and \\ M. Guainazzi \\ European Space Astronomy Centre of ESA, P.O. Box 78, Villanueva de la Canada, \\ E-28691 Madrid, SPAIN
}

\begin{abstract}
In this paper we study the nuclear obscuration of galaxies hosting Low Ionization Narrow Emission Regions (LINERs) based on their X-ray and optical emission. They show column densities at soft energies (0.5-2 keV) mostly related to the diffuse emission around the AGN, showing a correlation with the optical extinction. Column densities at hard energies $(2-10 \mathrm{keV})$ seem to be much higher than what would be expected from the optical extinction. They might be associated to the inner regions of the AGN, buried at optical wavelengths. The main result of this paper is that around $50 \%$ of our LINER sample shows signatures of Compton-thickness according to the most common tracers: the X-ray spectral index, $F_{X}(2-10 \mathrm{keV}) / \mathrm{F}([\mathrm{OIII}])$ ratio and FeK $\alpha$ equivalent width $(\mathrm{EW})$. However, the EWs of Compton-thick LINERs are significantly lower than in Compton-thick Seyferts $(\simeq 200 \mathrm{eV}$ against $\geq 500 \mathrm{eV})$, suggesting that the $2-10 \mathrm{keV}$ emission is dominated by electron scattering of the otherwise invisible AGN, or by emission
\end{abstract}

\footnotetext{
${ }^{1}$ Instituto de Astrofísica de Andalucía (CSIC), Granada, SPAIN
} 
from shocked gas associated to star formation rather than by reflection from the inner wall of the torus. However, no clear relation seems to exist between galaxies with optical dust lanes and X-ray classified Compton-thick objects. This may suggest that Compton-thick sources should be related to absorbing material located at the very inner regions of the AGN, maybe in the putative dusty torus. Larger black hole masses and lower Eddington ratios than Seyfert galaxies have been found. This effect can be better attributed to LINER nuclei being hosted by earlier morphological types than Seyfert nuclei. However, it has to be noted that, once a proper correction to the X-ray luminosity is applied, LINERs show Eddington ratios overlapping those of type 2 Seyferts. We speculate with a possible scenario for LINER nuclei: an inner obscuring matter similar to that of type 2 Seyfert, and an external obscuring matter responsible for the optical extinction. Compton-thick sources appear to be more common among LINERs than Seyferts.

Subject headings: LINERs - AGN - X-rays - Chandra - XMM-Newton.

\section{Introduction}

Active Galactic Nuclei (AGN) emit over the entire electromagnetic spectrum and are widely believed to be powered by the accretion of matter onto a super-massive black hole (SMBH, Rees 1984). Several families within the AGN category have been established from an observational point of view. Although their classification is sometimes misleading, it is widely believed that an unified model can explain them under a single scenario (Antonucci 1993). A key ingredient in this scheme is a dusty torus whose inclination with respect to the observer's line of sight is responsible for the dichotomy between optical type 1 (with broad permitted lines, face-on view) and type 2 (with narrow permitted lines, edge-on view) AGN. However, this scheme needs to be further confirmed because there are several sub-classes of objects that cannot be easily fitted into it. As an example, the nature of Narrow-line Seyfert

1 (Dewangan and Griffiths 2005) or non-obscured Seyfert 2 (Panessa and Bassani 2002) is still a matter of debate.

Low Ionization Nuclear Emission Line Regions (LINERs) are another sub-class of objects that cannot be easily included in the unified model. They are intriguing cases because, as suggested by their low X-ray luminosities $\left(\mathrm{L}(2-10 \mathrm{keV}) \sim 10^{39-42} \mathrm{erg} \mathrm{s}^{-1}\right)$, they could be the link between AGN $\left(\mathrm{L}(2-10 \mathrm{keV}) \sim 10^{41-45} \mathrm{erg} \mathrm{s}^{-1}\right)$ and normal galaxies (Zhang et al. 2009; Rovilos et al. 2009). Furthermore, they are the dominant population of active galaxies in the nearby universe (Ho et al. 1997; Ho 2008). Their signature in the optical spectrum 
is the enhancement of low ionization lines. However, this property alone is not enough to disentangle the nature of these galaxies because it can be explained by a variety of different physical processes (Ho 2008). Compact radio (Nagar et al. 2005) and hard X-ray cores (Gonzalez-Martin et al. 2009, and references therein, hereinafter GM+09) are the most secure signatures for the presence of an AGN. Although the AGN nature of a large number of LINERs has been confirmed from data at X-ray and radio frequencies, it is still unclear how LINERs do fit into the AGN unified scenario. A radiatively inefficient accretion flow onto the SMBH and/or a large amount of obscuring matter have been proposed as the main differences between LINERs and more luminous AGN (Dudik et al. 2009). X-rays are the ideal laboratory to test their nature, since they provide valuable information on both the obscuration and accretion rates.

We have analyzed the largest sample of LINERs up to now at X-ray frequencies (82 objects) with Chandra and XMM-Newton data (GM+09). Chandra's excellent angular resolution allowed us to investigate the X-ray nuclear properties of these galaxies (see also Gonzalez-Martin et al. 2006, hereinafter GM+06). According to their nuclear morphology in the $4.5-8 \mathrm{keV}$ band, we found that almost $60 \%$ of the sample shows an unresolved nuclear source, which is a clear hint of their AGN nature. The addition of XMM-Newton data offers us the opportunity to perform the spectral analysis on 60 out of the 82 objects. From the X-ray point of view, we concluded that LINERs are similar to type 2 Seyferts, both in luminosity and spectral shape (GM+09).

In this paper we discuss the properties and nature of the obscuring material covering LINER nuclei. In Section 2 we review the sample selection (already presented in GM+09); Section 3 presents the observational tracers of Compton-thick obscuration for our sample; in Section 4 we correlate the X-ray obscuration with other multiwavelength observables, to derive further clues on the origin of the obscuring material; and in Section 5 we discuss the implications of the obscuration on the LINER spectral energy distribution (SED) of LINERs. A summary of our results and the conclusions are provided in Section 6.

\section{The sample}

Our sample is presented in GM+09, where all the observational details are exhaustively explained. We briefly describe here the main characteristics of the sample.

The sample was extracted from the multi-wavelength LINER catalogue compiled by

Carrillo et al. (1999) (hereinafter MCL). The sample includes all the galaxies in MCL with available Chandra data up to 2007-06-30 and XMM-Newton data up to 2007-04-30. It 
includes 108 LINERs with Chandra data and 107 LINERs with XMM-Newton data. Seventy six objects are present in both archives yielding to a total of 139 LINERs.

LINER identifications were revised using Veilleux and Osterbrock (1987) diagnostic diagrams to discard out Seyfert, HII and transition objects. After optical re-identification we ended up with a final sample of 83 sources including 68 observed with Chandra and 55 with XMM-Newton. Observations for one of these objects showed strong pile-up effects leaving us with a final sample of 82 objects. Forty LINERs are found in both datasets. The final

sample mainly comprises objects from the Palomar Survey (Ho et al. 1997) and Luminous and Ultra-luminous IR galaxies (LIRGs and ULIRGs, mainly from Veilleux et al. 1999, and reference therein). Note that this sample is not complete because it comes from a catalogue containing all the known LINERs until 1999 and include only available data in Chandra and XMM-Newton archives.

See GM+09 for further details on the X-ray observations (its Sections 2 and 3 and Table 2), previously published X-ray data (its Appendix B), spectral fits used along this text (its Sections 4.2, 4.3 and 5.1.2), F-test statistic (its Tables 3 and 4), an example of spectral fit (its Fig. 4), spectral fit figures for Chandra and XMM-Newton data (in on-line format in its Appendix D and E), and final spectral fits (its Table 7).

\section{Compton-thickness}

The X-ray spectrum of LINERs can be described by two main components: (i) an absorbed primary power-law continuum; (ii) a soft spectrum (below $2 \mathrm{keV}$ ) described by an absorbed scattering plus/or a thermal component $(\mathrm{GM}+09)$. In this scenario, the column densities, called NH1 and NH2, provide information on the amount of absorbing material associated to the soft (0.5-2 keV) energy band and to the hard (2-10 keV) energy band, respectively.

If the X-ray obscuring matter has a column density which is equal to or larger than the inverse of the Thomson cross-section $\left(\mathrm{N}_{\mathrm{H}} \gtrsim 1.5 \times 10^{24} \mathrm{~cm}^{-2}\right)$, then the source is called, by definition, Compton-thick. If the X-ray obscuring matter has a column density lower than the Compton-thick limit but still in excess to the Galactic one the source is called Comptonthin. In Compton-thick AGN, the reflection components can be misinterpreted as a primary continuum and consequently induce a misclassification as Compton-thin or even unobscured AGN. Since the intrinsic continuum in Compton-thick sources is detectable at energies $>10$ $\mathrm{keV}$, only indirect proofs of the Compton-thick nature can be obtained with Chandra and XMM-Newton observations. 
From our data analysis, we found that NH2 covers a wide range of values $(\log (\mathrm{NH} 2)=$ $\left.20-24 \mathrm{~cm}^{-2}\right)$, the range covered by NH1 is much narrower, with a median value of $\log (\mathrm{NH} 1)$ $=21.32 \pm 0.71(\mathrm{GM}+09)$. Both column densities, NH1 and NH2, are given in Table 1 (Cols. 4 and 5). The reason for such a behavior might be related to the nature and location of the obscuration, as we discuss later. However, LINER nuclei can be Compton-thick sources and, in this case, the interpretation of the measured column density could be different.

The evaluation of Compton-thickness will be done based on three indirect diagnostics: (1) spectral index $(\Gamma<1),(2) \mathrm{F}_{\mathbf{X}}(2-10 \mathrm{keV}) / \mathrm{F}([\mathrm{OIII}])$ ratio $\left(\log \left(\mathrm{F}_{\mathbf{X}}(2-10 \mathrm{keV}) / \mathrm{F}([\mathrm{OIII}])\right)<0.5\right)$ and (3) high equivalent width of the neutral iron emission line $(\mathrm{EW}(\mathrm{FeK} \alpha)>500 \mathrm{eV})$. The relevant information of these three diagnostics and the classification according to them are reported in Table 2 ,

\subsection{Spectral index}

A flat spectrum above $\sim 2 \mathrm{keV}$ is one of the known Compton-thick diagnostics (Maiolino et al. 1998; Cappi et al. 2006). In addition to the models used in GM+09, in this paper we have fitted all the data to an absorbed power-law at energies larger than $2 \mathrm{keV}$ to have an independent measurement of the possible flat spectrum. Three Gaussian fits at 6.4, 6.7, and 6.95 $\mathrm{keV}$ have been included because FeK $\alpha$, FeXXVI and FeXXVII emission line are present in a number of objects $(\mathrm{GM}+09)$. The resulting spectral indices are listed in Table 2 (Col. 2). It should be noticed that for NGC 833, NGC 835, NGC 2639, UGC 4881, NGC 3507, NGC 3898, NGC 3945, MRK 266NE and NGC6482 a spectral fit was reported in GM+09 (more than 200 counts in $0.5-10 \mathrm{keV}$ energy band), but insufficient number counts above $2 \mathrm{keV}$ prevents their analysis here. Thus, this analysis was possible in 51 out of the 82 LINERs.

We consider a spectrum as flat when the resulting spectral index is consistent, within the uncertainties, with being smaller than 1.2 (Risaliti 2002; Beckmann et al. 2006; Dadina 2008; Winter et al. 2009). Statistically, flat spectra have been identified for 20 out of the 51 objects $(39 \%)$.

\section{2. $\mathrm{F}_{\mathrm{X}}(2-10 \mathrm{keV}) / \mathrm{F}([\mathrm{OIII}])$ ratio}

Maiolino and Rieke (1995) showed that the [OIII] line emission can be considered as a good isotropic indicator of the AGN power. On the other side, the 2-10 keV X-ray emission should be an intrinsic AGN property in the cases where the primary continuum is not suppressed by a highly obscuring material. When the primary continuum is suppressed 
due to heavy absorption $\left(\mathrm{N}_{\mathrm{H}}>10^{24} \mathrm{~cm}^{-2}\right)$, the ratio between hard X-rays and [OIII] line emission lowers because the computed X-ray luminosity is underestimated. For a large sample of type 2 Seyferts, the $\log \left(\mathrm{F}_{\mathrm{X}}(2-10 \mathrm{keV}) / \mathrm{F}([\mathrm{OIII}])\right)$ ratio has been used as a good diagnostic to discriminate between Compton-thick and Compton-thin sources (Bassani et al. 1999; Panessa and Bassani 2002; Maiolino et al. 2003; Panessa et al. 2005, 2006). We will use this criterion to search for Compton-thick LINER nuclei.

We have searched in the literature for [OIII] emission line fluxes and $\mathrm{H} \alpha / \mathrm{H} \beta$ ratios. Data are available for 79 out of the 82 objects (except for NGC 835, CGCG 162-010 and IC 1459). The optical extinction is computed using $A v=6.67 \times \log (f(H \alpha) / R v \times f(H \beta))$. Whenever the Balmer Decrement is lower than 3.1, we assume a value of 3.1, corresponding to zero optical extinction (Osterbrock 1987). $\mathrm{L}(2-10 \mathrm{keV})$ is taken from GM+09. Reddening-corrected [OIII] emission line fluxes and optical extinctions are reported in Table 1 (Cols. 7 and 8) and the $\log \left(F_{X}(2-10 \mathrm{keV}) / F([\mathrm{OIII}])\right)$ ratios are shown in Table 2 (Col. 4).

Fig. 1] shows the histogram of $\log \left(\mathrm{F}_{\mathrm{X}}(2-10 \mathrm{keV}) / \mathrm{F}([\mathrm{OIII}])\right)$ ratios. We have fitted the distribution to a two Gaussian model (see continuous and dashed lines in Fig. 1). The double Gaussian model are centred at $\log \left(\mathrm{F}_{\mathrm{X}}(2-10 \mathrm{keV}) / \mathrm{F}([\mathrm{OIII}])\right)_{\mathrm{o} 1}=-0.24$ and $\log \left(\mathrm{F}_{\mathrm{X}}(2-10 \mathrm{keV})\right.$ $/ \mathrm{F}([\mathrm{OIII}]))_{\mathrm{o} 2}=1.39$, with $\sigma=0.6$ and $\sigma=0.3$, respectively. The minimum between the two Gaussian fits occurs at $\log \left(\mathrm{F}_{\mathrm{X}}(2-10 \mathrm{keV}) / \mathrm{F}([\mathrm{OIII}])\right)=0.68$. The distribution is similar to that found by Maiolino et al. (1998) for Seyfert galaxies. It shows two peaks centered at $\log \left(\mathrm{F}_{\mathrm{X}}(2-10 \mathrm{keV}) / \mathrm{F}([\mathrm{OIII}])\right)=-0.5$ and $\log \left(\mathrm{F}_{\mathrm{X}}(2-10 \mathrm{keV}) / \mathrm{F}([\mathrm{OIII}])\right)=1.0$, with the later value corresponding to the average ratio found for type 1 Seyfert galaxies and the former to that of Compton-thick objects. Note that the Gaussian fit is centred at the Compton-thick regime $\left(\log \left(\mathrm{F}_{\mathrm{X}}(2-10 \mathrm{keV}) / \mathrm{F}([\mathrm{OIII}])\right)_{\mathrm{o} 1}=-0.24\right)$ shows an area that represents the $63 \%$ of the sample.

We have decided to define two regimes to ensure the robustness of our classification: (1) $\log \left(\mathrm{F}_{\mathrm{X}}(2-10 \mathrm{keV}) / \mathrm{F}([\mathrm{OIII}])\right)<0$ and $(2) 0<\log \left(\mathrm{F}_{\mathbf{X}}(2-10 \mathrm{keV}) / \mathrm{F}([\mathrm{OIII}])\right)<0.5$. The value $\log \left(\mathrm{F}_{\mathrm{X}}(2-10 \mathrm{keV}) / \mathrm{F}([\mathrm{OIII}])\right)<0.5$ corresponds to the limit reported by Maiolino et al. (1998) between type 1 Seyferts and Compton-thick type 2 Seyferts. The ratio $\log \left(\mathrm{F}_{\mathrm{X}}(2-10 \mathrm{keV}) / \mathrm{F}([\mathrm{OIII}])\right)$ is the conservative limit assumed by Bassani et al. (1999).

Maiolino et al. (1998) presented a sample of 8 heavily absorbed type 2 Seyferts by means of BeppoSAX data. They measured their column densities, spectral index and $\mathrm{EW}(\mathrm{FeK} \alpha)$, classifying all of them as Compton-thick sources. They compared the ratio $\log \left(F_{X}(2-10 \mathrm{keV}) / F([\mathrm{OIII}])\right)$ with that of type 1 Seyferts by Mulchaey et al. (1994). This ratio is $\log \left(\mathrm{F}_{\mathrm{X}}(2-10 \mathrm{keV}) / \mathrm{F}([\mathrm{OIII}])\right) \sim 1$ for type 1 Seyferts while Compton-thick sources always show $\log \left(\mathrm{F}_{X}(2-10 \mathrm{keV}) / \mathrm{F}([\mathrm{OIII}])\right)<0.5$ (see Fig. 3 in Maiolino et al. 1998). On the other hand, Bassani et al. (1999) compared the column density versus the ratio 
$\log \left(F_{X}(2-10 \mathrm{keV}) / F([\mathrm{OIII}])\right)$ for 72 type 2 Seyferts. They also refered to Mulchaey et al. (1994) for type 1 Seyferts. They found a ratio $\log \left(\mathrm{F}_{\mathrm{X}}(2-10 \mathrm{keV}) / \mathrm{F}([\mathrm{OIII}])\right)=0$ for heavily absorbed sources. However, they compile their sample using all the type 2 Seyferts observed in the 2-10 keV range. To establish the limit between Compton-thick and Compton-thin sources, we consider crucial that column densities of Compton-thick sources must be confirmed above $10 \mathrm{keV}$. Thus, we have used the limit given by Maiolino et al. (1998), since they used BeppoSAX data above $10 \mathrm{keV}$.

To validate such a limit we have compared the distribution of $\log \left(\mathrm{F}_{\mathbf{X}}(2-10 \mathrm{keV}) / \mathrm{F}([\mathrm{OIIII}])\right)$ (see Fig. 2) obtained for our LINER sample with that for unobscured PG QSOs (Jimenez-Bailon et al. 2005; Piconcelli et al. 2005) and Compton-thick sources reported by (Bassani et al. 1999). The [OIII] fluxes of PG QSOs are taken from Marziani et al. (2003) and corrected for reddening assuming $\mathrm{H} \alpha / \mathrm{H} \beta=4.5$ (mean value for QSOs by York et al. 2006). Unobscured QSOs show $\log \left(\mathrm{F}_{\mathrm{X}}(2-10 \mathrm{keV}) / \mathrm{F}([\mathrm{OIII}])\right)>0$., in agreement with our second peak centred at $\log \left(\mathrm{F}_{\mathrm{X}}(2-10 \mathrm{keV}) / \mathrm{F}([\mathrm{OIII}])\right)_{\circ 2}=1.39$. Compton-thick AGN fall in the region of Comptonthick LINERs. Moreover, Lamastra et al. (2009) have recently shown that Compton-thin type 2 Seyferts range between $0<\log \left(\mathrm{F}_{\mathrm{X}}(2-10 \mathrm{keV}) / \mathrm{F}([\mathrm{OIII}])\right)<3$, overlapping with the PG-QSOs.

Thirty three out of the 79 LINER galaxies $(42 \%)$ have $\log \left(\mathrm{F}_{\mathbf{X}}(2-10 \mathrm{keV}) / \mathrm{F}([\mathrm{OIII}])\right)<0$ following the more conservative criterion. Nine of them show ratios $0<\log \left(F_{X}(2-10 \mathrm{keV})\right.$ $/ F([\mathrm{OIII}]))<0.5$. The fraction of LINERs with $\log \left(\mathrm{F}_{\mathrm{X}}(2-10 \mathrm{keV}) / \mathrm{F}([\mathrm{OIII}])\right)<0.5$ is then $53 \%(42 / 79)$.

Two points must be stressed on the use of this diagnostic to find Compton-thick objects. First, we notice that 21 sources have an unrealistic ratio of $\mathrm{H} \alpha / \mathrm{H} \beta$ below 3.10, the assumed ratio for AGN (Osterbrock and Ferland 2006). This might be due to a problematic continuum subtraction. Therefore in these 21 cases we have assumed a minimum value of 3.1, which means that no correction has been performed. The [OIII] emission line flux hence is underestimated, and therefore the number of Compton-thick LINERs in our sample can be taken as a lower limit.

The second comment is about the eventual contamination by emission from the host galaxy and/or circumnuclear environment. Objects with a disrupted morphology, like merging galaxies, could have an enhancement of star formation processes that over-shine the host galaxy emission. The usage of the [OIII] emission lines versus X-ray luminosity ratio as a Compton-thick indicator could lead to misleading results. A large fraction of the measured [OIII] emission line flux could come from star-forming processes and the nuclear [OIII] emission line luminosity could be overestimated. 
LIRGS is well known that they show perturbed morphologies and an enhancement of star formation which can turn out in large values of [OIII] fluxes. Evidence of perturbed morphologies is present in only 7 cases (IIIZW 035, UGC 4881, NGC 3690B, IRAS 12112+0305, NGC 4410A, MRK 266NE and NGC6240, see GM+091), all of them classified according to this criterion as Compton-thick candidates. NGC 3690B and NGC 6240 are well known Compton-thick sources (see Section 3.1). The remaining 5 cases should be taken with reservation.

A contamination of the [OIII] flux measurement by merging-driven star formation should affect more significantly galaxies at a larger distance, because a larger fraction of the morphological disturbances are included in the spectroscopic aperture. However, the fraction of under [OIII]-luminous LINERs in our sample does not depend on the source distance. Using objects with the smallest distance $(\mathrm{D}<50 \mathrm{Mpc})$ we get the same percentage: 27 out of the 54 objects $(50$ per cent $)$ show $\log \left(\mathrm{F}_{\mathrm{X}}(2-10 \mathrm{keV}) / \mathrm{F}([\mathrm{OIII}])\right)<0.5$. In fact, no trend is found between this ratio and the distance (correlation coefficient $r=0.15$ ). Therefore, aperture effects can be ruled out.

Among unperturbed morphologies, evidences of circumnuclear star formation have been found in only three cases: NGC 3507, NGC 3998 and NGC 4321 (Gonzalez-Delgado et al.

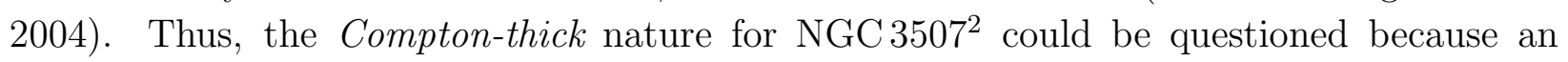
enhancement of the [OIII] line emission might be expected due to the ionized emission in the central region. Recently, Walsh et al. (2008) discard such a possibility based on STIS/HST data: the $\mathrm{H} \alpha+[\mathrm{NII}]$ emission line morphology appears to be very compact with no extended features attributable to circumnuclear star forming events.

Furthermore, Ho (2008) remarked that all the classes of LLAGN (Seyfert, LINERs and Transition objects) show the same host galaxy properties after a carefully decontamination of the differences coming from the Hubble type distribution of each class. Only transition objects seem to show a mild enhancement of star formation of the host galaxy. This is also the case for their circumnuclear environments. On nuclear scales smaller than 10pc, Sarzi et al. (2005) studied the stellar population of nearby LLAGN, finding that only 1 out of their 4 LINERs showed young stellar populations. Gonzalez-Delgado et al. (2004, 2008a) found also that LINERs host old stellar population. Thus, recent star-formation does not seem to be an important ingredient in LINERs. Therefore, in general terms we could expect that the [OIII] to X-ray flux ratio would be as good tracer as already previous studies have shown for Seyfert nuclei (Maiolino et al. 1998; Cappi et al. 1999; Bassani et al. 2000;

\footnotetext{
${ }^{1}$ The post stamps of DSS images at $150 \mathrm{kpc}$ scale are provided in Appendix $\mathrm{F}$ in GM+09.

${ }^{2}$ The only case among the three galaxies with $\left.\log \left(\mathrm{F}_{\mathrm{X}}(2-10 \mathrm{keV}) / \mathrm{F}([\mathrm{OIII}])\right)<0.5\right)$
} 
Panessa et al. (2006); Cappi et al. 2006). This result is reinforced because Seyfert 1, Seyfert 2 and unboscured QSOs follow the same relation between 2-10 keV X-ray luminosity and [OIII] emission line luminosity than LINER nuclei (see Section 4.7).

\subsection{EW of the neutral iron emission line}

Leahy and Creighton (1993) found that $\mathrm{EW}(\mathrm{FeK} \alpha)$ is another Compton-thick tracer. The idea comes from the finding that $\operatorname{EW}(\mathrm{FeK} \alpha)$ for Seyfert 1 galaxies typically amount to a few hundred eVs (Turner et al. 1998; Perola et al. 2002; Panessa et al. 2008). When the column density increases to a few $10^{23} \mathrm{~cm}^{-2}$, EWs increase, because they are measured against a suppressed continuum. EW $(\mathrm{FeK} \alpha)$ can reach values as high as $500 \mathrm{eV}$ for column densities larger than $10^{24} \mathrm{~cm}^{-2}$ (Matt 1997; Bassani et al. 1999).

For our sample of 82 LINERs, clear detections of FeK $\alpha$ lines are found in 10 galaxies with XMM-Newton data and in 7 galaxies with Chandra data, 4 of them in common (see Gonzalez-Martin et al. 2008). All together we have positive detection for NGC 0315, NGC 0833, NGC 0835, NGC 1052, UGC 05101, NGC 3690B, NGC 4486, NGC 4579, MRK 0266NE, UGC 08696, NGC 6240, NGC 7130 and NGC 7285. Their EW and uncertainties are presented in Table 2, Only three out of the 13 detected FeK $\alpha$ line show EW>500 eV.

In order to further explore the use of $\operatorname{EW}(F e K \alpha)$ as a tracer for obscuration, we analyze whether its value depends on the absorbing column density NH2 (Fig. 3). Upper limits for column densities were excluded from this analysis. The trend that we find is consistent with the predictions by Ghisellini et al. (1994) 3 .

To validate the calculated values, we have also checked that they do not depend on the choice of the X-ray spectral continuum. We have used two physical models to fit the underlying continuum in the Compton-thick scenario: (1) A single reflection model to the hard X-rays ( $>2 \mathrm{keV}$ ) (PEXRAV in Xspec, Magdziarz and Zdziarski 1995) and (2) the baseline model for Compton-thick sources reported by Guainazzi et al. (2005). Because of the LINER complex spectrum below $2 \mathrm{keV}$ (fitted by thermal and/or power-law components), the first model could add complementary information using only the 2-10 keV energy range.

We have used a pure reflection model by fixing the reflection scaling factor to -1 and solar abundances. The power-law spectral index is linked to the intrinsic continuum powerlaw in the second model. The fit is performed for nuclei with more than 200 counts in

\footnotetext{
${ }^{3}$ Below $10^{23} \mathrm{~cm}^{-2}$, EW $($ FeK $\alpha)$ seem to remain constant with values around $100 \mathrm{eV}$ and for $\mathrm{NH} 2>10^{23} \mathrm{~cm}^{-2}$, $\mathrm{EW}(\mathrm{FeK} \alpha)$ appear to increase up to $\sim 500 \mathrm{eV}$ at $10^{24} \mathrm{~cm}^{-2}$.
} 
the band where the fit is made (i.e. $2-10 \mathrm{keV}$ and $0.5-10 \mathrm{keV}$, respectively). The resulting $\mathrm{EW}(\mathrm{FeK} \alpha)$ are presented in Table 2 (Col. 7 and 8).

In general, the EWs obtained using the best-fit reported by GM+09 are confirmed with these two models. Using the single reflection model above $2 \mathrm{keV}$ and taking upper limits, NGC 410 changes to $\mathrm{EW}(\mathrm{FeK} \alpha)>500 \mathrm{eV}$ and NGC 4374 changes to $\mathrm{EW}(\mathrm{FeK} \alpha)<500 \mathrm{eV}$. Using the baseline model for Compton-thick sources, NGC 0833 changes to EW(FeK $\alpha)<$ $500 \mathrm{eV}$ and UGC 05101 and NGC 6240 show an upper value slightly higher than $500 \mathrm{eV}$ (510 and $530 \mathrm{eV}$, respectively).

\section{Discussion}

\subsection{Previously found Compton-thick LINERs in our sample}

Based on BeppoSAX data, sensitive above $10 \mathrm{keV}$ up to $300 \mathrm{keV}$, two of the LINERs in the sample, NGC 3690B, and NGC 6240, show intrinsic continuum above $10 \mathrm{keV}$, typical of Compton-thick sources (Ptak et al. 2003; Ceca et al. 2002; Risaliti et al. 1999; Vignali et al. 1999). UGC 05101 and NGC 5005 have been also claimed to be Compton-thick by using an indirect method. Based on the detection of a high upper limit for the $\operatorname{EW}(\mathrm{FeK} \alpha)$ on $A S C A$ data Risaliti et al. (1999) claimed a column density for NGC 5005 larger than $10^{24} \mathrm{~cm}^{-3}$. With more recent XMM-Newton data, Guainazzi et al. (2005) questioned its Compton-thick nature. A highly obscured nuclei $\left(\mathrm{NH} \sim 10^{24} \mathrm{~cm}^{-3}\right)$ has been reported by Imanishi et al. (2003) for UGC 05101. Moreover, recently Teng et al. (2008) have classified UGC 08696 as a Compton-thick source, using Suzaku data above $10 \mathrm{keV}$. However, we have decided not to use it as Compton-thick source because they have reported changes in the spectral shape that might be due to large changes in the absorbing column density. Hereinafter we consider UGC 05101, NGC 3690B, and NGC 6240 objects as Compton-thick sources according to published literature results (hereinafter we refer to them as 'Confirmed Compton-thick'). For the remaining nuclei in our sample, no signs of a Compton-thick nature have been reported in the literature.

Concerning the indirect Compton-thick diagnostics used above, two of the confirmed Compton-thick show flat spectra (UGC 05101 and NGC 6240) whereas NGC 3690B seems to have a somewhat steeper spectrum. Thus, a classification of Compton-thickness based solely in this criterion needs to be taken with some caution. Moreover, the three confirmed Compton-thick show $\log \left(\mathrm{F}_{\mathrm{X}}(2-10 \mathrm{keV}) / \mathrm{F}([\mathrm{OIII}])\right)<0$, consistent with being Compton-thick object. Also, we have been able to determine the $\operatorname{EW}(\mathrm{FeK} \alpha)$ of the three confirmed Comptonthick in our sample, finding $\mathrm{EW}(\mathrm{FeK} \alpha)=280 \pm 180 \mathrm{keV}, \mathrm{EW}(\mathrm{FeK} \alpha)=230 \pm 110 \mathrm{keV}$ and 
$\mathrm{EW}(\mathrm{FeK} \alpha)=380 \pm 60 \mathrm{keV}$, for UGC 05101, NGC 3690B and NGC 6240 respectively (shown as black stars in Figs. 3 and 4 . These values are compatible with the values $410 \pm 250 \mathrm{eV}$, $420 \pm 260 \mathrm{eV}$ and $300 \pm 100 \mathrm{eV}$ reported in the literature for UGC 05101 (Imanishi et al. 2003), NGC 3690B (Ballo et al. 2004) and NGC 6240 (Boller et al. 2003), respectively. Our values do not agree with those expected for Compton-thick sources $(\mathrm{EW}(\mathrm{FeK} \alpha)>500 \mathrm{eV})$. When the baseline model for Compton-thick sources is used, the values for UGC 05101 and NGC 6240 raise to 510 and $530 \mathrm{eV}$, respectively (although the fit is statistically worse than for the best fit model). We discuss the use of a low $\mathrm{EW}(\mathrm{FeK} \alpha)$ for Compton-thickness diagnostics in Section 4.3 .

\subsection{Compton-thick LINERs}

Table 2 shows the Compton-thick classification following the three tracers. Cols. 3, 5 and 9 of Table 2 show the Compton-thick classification according to the three diagnostics and Col. 10 gives the final Compton-thick classification. 'CT' indicates Compton-thick classified, '?' not available information and 'CT?' possible Compton-thick source.

We have information on at least one of the tracers for the whole sample. We have defined a source to be Compton-thick when at least one tracer indicates such a classification and none of the others contradict it. When two tracers agree we have taken this classification, even if the third diagnostic does not agree. We have classified as 'CT?' four objects with only two tracers available showing contradicting results (NGC 0833, NGC 3690B, NGC 5005, MRK 266NE). However, note that NGC 3690B is a confirmed Compton-thick (see Sect. 4.1).

Adding the information coming from the three Compton-thick tracers, Compton-thickness very often appears among LINERs, representing 49\% (40/82) of our sample (54\% including 'CT?'). Furthermore, at least one of the tracers indicates consistency with a Compton-thick nature in $62 \%$ of our sample.

Cappi et al. (2006) reported a sub-sample of 27 optically-selected and distance-limited Seyfert galaxies $\left(\mathrm{F}(2-10 \mathrm{keV})>10^{13} \mathrm{erg} \mathrm{s}^{-1} \mathrm{~cm}^{-2}\right), 5$ of them being Compton-thick (18\%). However, four of their objects are in our LINER sample (NGC 2685, NGC 3185, NGC 4579 and NGC 4698). Excluding these four objects, 4 out of the remaining 23 objects are classified as Compton-thick candidates (17\%). An extension of this sample was studied by Panessa et al. (2006) (without distance completeness of the sample), founding arguments favouring the Compton-thickness nature in 11 out of their 47 Seyfert (23\%) galaxies; however, among them 10 objects are LINERs (NGC 2639, NGC 2655, NGC 2685, NGC 3185, NGC 3608, NGC 3627, NGC 4579, NGC 4698, NGC 6482 and NGC 7743). Excluding these 
objects, 9 out of the 37 Seyfert galaxies in Panessa et al. (2006) are Compton-thick sources (24\%). For the 10 objects in common, they classified NGC 3185 and NGC 7743 as Comptonthick objects, in agreement with our classification. However, we also classify as such NGC 2639, NGC 2685, NGC 3608, NGC 3627 and NGC 4698. Note that their classification is based on the $F_{X}(2-10 \mathrm{keV}) / F([\mathrm{OIII}])$ versus $\mathrm{F}_{\mathrm{IR}} / \mathrm{F}([\mathrm{OIII}])$ diagram. All these 5 objects show a $\log \left(\mathrm{F}_{\mathrm{X}}(2-10 \mathrm{keV}) / \mathrm{F}([\mathrm{OIII}])\right)<0$ except NGC 2639, that they classified as a possible Comptonthick object. Closer to our findings, Guainazzi et al. (2005) found a $46 \%$ Compton-thick sources in a sample of 49 nearby Seyfert galaxies (40\% if we exclude sources in our LINER sample). Thus, Compton-thick objects seems to be more frequently found in LINERs than in type 2 Seyferts.

\subsection{Low $\operatorname{EW}(F e K \alpha)$ Compton-thick sources}

We have explored the validity of the $\operatorname{EW}(\mathrm{FeK} \alpha)$ as a Compton-thick tracer studying the connection between the $\mathrm{EW}(\mathrm{FeK} \alpha)$ and the NH2 column density (see Fig. 3). Below $10^{23} \mathrm{~cm}^{-2}$, the EW $(F e K \alpha)$ of Seyfert 1 nuclei (Nandra et al. 2007) and unobscured quasars (Jimenez-Bailon et al. 2005; Piconcelli et al. 2005) seem to remain constant around $100 \mathrm{eV}$. For $\mathrm{NH} 2 \gtrsim 10^{23} \mathrm{~cm}^{-2}$, the $\mathrm{EW}(\mathrm{FeK} \alpha)$ seems to increase up to $\sim 500 \mathrm{eV}$ at $10^{24} \mathrm{~cm}^{-2}$ for a sample of 49 type 2 Seyferts (Guainazzi et al. 2005). Our trend does not seem to be inconsistent with this behaviour although better constraints on the EW, perhaps with new observations, would be required in order to make any conclusion. The similarities of this trend with that reported by Guainazzi et al. (2005) would manifest that LINERs might be sharing the same origin than Seyfert galaxies for the iron line, which is originated in the inner wall of the torus (Ghisellini et al. 1994).

Moreover, we have found a mismatch between the $\mathrm{EW}(\mathrm{FeK} \alpha)$ and $\log \left(\mathrm{F}_{\mathrm{X}} / \mathrm{F}[\mathrm{OIII}]\right)$ diagnostics. This is clearly seen in Fig. 4. The correlation between these two quantities was used as a Compton-thick diagnostic by Bassani et al. (1999). They found that both, $\mathrm{EW}(\mathrm{FeK} \alpha)$ and column density, decrease when the ratio $\mathrm{F}_{\mathrm{X}}(2-10 \mathrm{keV}) / \mathrm{F}([\mathrm{OIII}])$ increases (region filled with red horizontal lines in Fig,4). They found $F_{X}(2-10 \mathrm{keV}) / F([\mathrm{OIII}])>0$ and $\mathrm{EW}(\mathrm{FeK} \alpha) \sim 100 \mathrm{eV}$ for type 1 Seyfert galaxies (square filled with diagonal black square), whereas Compton-thick galaxies were located at $\mathrm{EW}(\mathrm{FeK} \alpha)>500 \mathrm{eV}$ and $\mathrm{F}_{\mathrm{X}}(2-10 \mathrm{keV})$ $/ \mathrm{F}([\mathrm{OIII}])<0$ (red continuous line).

At least half of our sample is located in the region occupied by type 1 Seyferts. However, neither of the confirmed Compton-thick sources (see Sect. 4.1) fall in the expected region. Two of them marginally fall in this regime when the baseline model for Compton-thick AGN is used. We notice that in order to include Compton-thick objects, the EW(FeK $\alpha)$ 
limit needs to be re-defined to a lower value of EW(FeK $\alpha) \sim 200 \mathrm{eV}$. NGC 2681, UGC 05101, NGC 3690B, NGC 4374, NGC 4410A, MRK 266NE, NGC 5363, NGC 6240, IRAS 17208-0014 and NGC 7130 fall in this regime. However, such a low limit on EW(FeK $\alpha)$ does not allow to distinguish between type 1 Seyferts and Compton-thick sources with low $\mathrm{F}_{\mathbf{X}}(2-10 \mathrm{keV})$ $/ \mathrm{F}([\mathrm{OIII}])$.

Therefore, it seems that a new population of Compton-thick sources with low EW(FeK $\alpha)$ emerges. The same result has been claimed by Brightman and Nandra (2008). They explain their finding by postulating that the X-ray emission is dominated by scattering of the otherwise invisible obscured AGN emission, rather than by reflection from the inner wall of the torus.

Compton-thick LINERs are mostly fitted with a combination of a thermal plus a powerlaw model. The later can be interpreted as the primary continuum or as the scattering component. Thus, the hard continuum can be completely lost under the scattering continuum. However, the scattering component contributes always less than $10 \%$ of the fluxes above $2 \mathrm{keV}$ for Compton-thick candidates. In LINERs, the thermal emission could be also responsible for the decreasing of the $\operatorname{EW}(\mathrm{FeK} \alpha)$ because a strong contribution of this component is found in a large number of them (see GM+09).

\subsection{Origin of the obscuring material}

In Seyfert 2 galaxies, the NH2 column density is related to the obscuration of the primary continuum (Bianchi et al. 2004; Guainazzi et al. 2005; Panessa et al. 2006; Cappi et al. 2006). This absorption in LINERs covers a range compatible with them. It does not appear to be the case for NH1 column density: consistent with the Galactic value in Seyferts (Bianchi et al., 2009), NH1 column density in LINERs is an order of magnitude above the Galactic value. The fact that our column density is larger than that expected for Seyfert galaxies has opened the question of its origin. Two possible origins arise: (1) material very close to the nucleus; (2) material in the host galaxies, with a column density larger than the expected for the Galactic value.

We have made the spectral analysis of the diffuse emission around the nucleus using Chandra data to investigate the origin of the NH1 column density. From the sample of 55 objects with spectral fitting, we have selected the 19 objects for which the spectral analysis on the diffuse emission is expected to be reliable (see GM+09 for a detailed explanation of the diffuse emission analysis).

The comparison between the nuclear NH1 column densities (NH1(nucleus)) and the 
diffuse emission NH1 column densities (NH1(diffuse emission)) is shown in Fig. 5. For six galaxies (NGC 4125, NGC 4321, NGC 4374, NGC 4552, NGC 4696 and IC 1459) only upper limits for NH1(diffuse emission) have been obtained. In six galaxies (NGC 0315, 3C 218, NGC 4111, NGC 4261, NGC 4579 and CGCG 162-010) NH1(diffuse emission) is compatible with the Galactic value, whereas NH1(nucleus) is larger. Thus, it can be safely concluded that in these cases the material responsible for NH1 column density is unrelated to the host galaxy, and must be located in the nuclear region. For NGC 4278, NH1(diffuse emission) is larger than NH1(nucleus). For the remaining 6 galaxies, nuclear and diffuse emission NH1 column densities lie, within the errors, in the unity slope line, what can be interpreted as both having the same origin, i.e. most probably related to the material in the host galaxy. In order to establish whether this is due to a dustier host galaxy environment in LINERs than in Seyferts, we discuss in the next subsection the optical Balmer decrement distribution in our LINER sample.

\subsection{X-ray versus optical obscuration}

In this section we investigate the relation between the X-ray obscuring material and the optical extinction, Av. To do so, we have computed the optical extinction by using the $\mathrm{H} \alpha / \mathrm{H} \beta$ ratios. Objects showing the lower limit $(\mathrm{H} \alpha / \mathrm{H} \beta=3.10)$ have been removed from this analysis. Assuming a Galactic ratio $A v / N_{H}=5 \times 10^{-22} \mathrm{~cm}^{-2}$, we can express Av in units of $\mathrm{cm}^{-2}$. Fig. [6] shows the histograms of the ratio between NH1 and Av (Left) and NH2 and Av (Right). While NH1 column density seems to be related to the optical extinction, NH2 appears to be much larger. This result was already drawn by Maiolino (2001) for a sample of type 2 Seyfert galaxies. In their sample of unobscured type 2 Seyferts, Panessa and Bassani (2002) found that the obscuration measured by the column densities at X-ray frequencies is consistent with that at the optical wavelengths $(\mathrm{Av})$. This appears to be in good agreement with our results, since the column density measured by Panessa and Bassani (2002) is more related to NH1 than to NH2 since they use only one absorption.

Interestingly, the overwhelming majority of LINERs in our sample (59 out of 67) show compact nuclear sources in HST (see Col. 9 of Table 1) sharp-divided images (see GM+06 for methodology explanation). This is consistent with optical HST data recently published by Gonzalez-Delgado et al. (2008b) for a sample of LINERs and Transition Objects; we have found that only 5 out of their 34 LINERs appear not to be compact. This rules out association of the optical dust line to the material responsible for NH1 column density.

\footnotetext{
${ }^{4} H S T$ images, mainly with the filter F814W, can be seen in the Appendix C (panel G) and GM+09.
} 
Objects with a dusty environment (coded as 'D' in Table 1) are equally distributed between Compton-thin and Compton-thick sources. However, this result must be taken with some reserves due to the low statistics. Guainazzi et al. (2001), in contrast to our result, found that Compton-thin Seyferts prefer dustier environment whereas Compton-thick Seyferts distribute both in dustier and dust-free regions. Based in the present data a relationship between the optical morphology and Compton-thickness is not evident, being LINERs not specially dustier objects.

\subsection{Obscuration and environment}

To take into account the eventual influence of the environmental status in the properties of our sample galaxies, we have searched in NED for possible companions at projected distances smaller than $250 \mathrm{kpc}$. Galaxies have been classified into 4 groups according to their environment: 1) isolated, when no projected companions with comparable sizes and

redshift difference smaller than $1000 \mathrm{~km} / \mathrm{s}$ are found, 2) pairs, when only another galaxy of comparable size and close in redshift is identified (wide and close pairs together with merging systems are grouped into this category), 3) groups, for galaxies in small group environments (either compact or loose), 4) clusters, for galaxies known to reside in cluster environments (in some cases, they correspond to the cluster center, see GM+09).

The environmental status does not seem to be connected with any of the analyzed properties, except the Compton-thickness. The resulting average and sigma values of $\mathrm{L}(2-10 \mathrm{keV})$ $/ \mathrm{L}([\mathrm{OIII}])$ for the different environments are provided in Table 3 , and the corresponding histograms shown in Fig. 7. We note that Compton-thick objects seem to be frequent in cluster environments (14\%), but are relatively frequent in groups (67\%), pairs and merging systems (65\%). The probability that cluster and group distributions are the same is $19 \%$.

This result could be understood by considering that a huge amount of obscuring material is being transported to the nuclear regions as a result of the merging process (for instance Mihos and Hernquist 1994; Barnes and Hernquist 1996). In fact, for ULIRGs, galaxy mergers are invoked to produce massive in-falls of gaseous material towards their centers (e.g. Rupke et al. 2008) and column densities $\geq 10^{24} \mathrm{~cm}^{-2}$ have been deduced from CO measurements (e.g. Downes and Solomon 1998; Evans et al. 2002).

Nevertheless, a number of galaxies in the group of isolated objects appear also as Compton-thick candidates (NGC 2639, NGC 2685, NGC 4457, NGC 4698, NGC 7331 and NGC 7743). The origin of the obscuring material in these systems has to be related either to intrinsic properties or to secular processes in their host galaxies. 
The statistical results are not conclusive and a bigger sample would be needed with a better coverage of the different environments. However, the results are suggestive of a connection between the environment and the Compton-thickness. Krongold et al. (2003) found that type 2 Seyferts and type 2 LINERs show the same rate of companions in the local universe, while type 1 Seyferts show a lower rate of companions. However, recently Montero-Dorta et al. (2008) have found that high redshift LINERs in DEEP2 tend to favour higher density environments while Seyfert do not show environmental dependencies.

If the fraction of Compton-thick LINERs is dependent on the environment, a large population of Compton-thick sources might be existing in the high redshift Universe. This is the case of IRAS 00182-7112, an optically classified LINER nucleus at redshift 0.3. The FeK $\alpha$ emission line is not present, consistent with our nearby LINER sample, but a high EW of the He-like Fe $\mathrm{K}$ line and a flat spectrum indicate that the source is reflection-dominated (Nandra and Iwasawa 2007). Unfortunately, this population is probably missed with the current instrumentation, due to their low luminosity.

\subsection{Obscuration and Eddington ratios}

Several authors have pointed out that the difference between LINERs and Seyferts comes from their Eddington ratios being smaller in LINERs (Dudik et al. 2005; Ho 2008). Here we include the Compton-thick nature of the sources into the analysis. We will demonstrate the importance of their effect in the estimation of the Eddington rate.

Assuming that all the candidates are actually Compton-thick, their intrinsic column densities should be higher than $10^{24} \mathrm{~cm}^{-2}$. Hence, their X-ray luminosities are underestimated since the applied column density correction is lower than the real. To estimate the correction factor for Compton-thick sources we divided our objects in two groups with $\mathrm{F}_{\mathbf{X}}$ $/ \mathrm{F}([\mathrm{OIII}])>0.5$ (Compton-thin) and $\mathrm{F}_{\mathrm{X}} / \mathrm{F}([\mathrm{OIII}])<0.5$ (Compton-thick), respectively. The median values are $\left.<\mathrm{F}_{\mathrm{X}} / \mathrm{F}([\mathrm{OIII}])_{\text {thin }}\right\rangle=16.5$ and $<\mathrm{F}_{\mathrm{X}} / \mathrm{F}([\mathrm{OIII}])_{\text {thick }}>=0.4$. We use the ratio between these two quantities as the correction factor between Compton-thick and Compton-thin sources: $<\mathrm{F}_{\mathrm{X}}(2-10 \mathrm{keV}) / \mathrm{F}([\mathrm{OIII}])_{\text {thin }}>/<\mathrm{F}_{\mathrm{X}}(2-10 \mathrm{keV}) / \mathrm{F}([\mathrm{OIII}])_{\text {thick }}>$ $=41.45$. Applying this correction factor to our Compton-thick sources we can obtain the corresponding corrected X-ray luminosities (reported in Table1, Col. 6). The median value and standard deviation of the hard X-ray luminosity and of the ratio between the Compton-thick corrected $(2-10 \mathrm{keV})$ and $[\mathrm{OIII}]$ emission line luminosities is now $\log (\mathrm{L}([\mathrm{OIII}]))=40.5 \pm 1.4$

\footnotetext{
${ }^{5}$ Note that the same method was applied by Panessa et al. (2006) to correct Compton-thick Seyferts, using as the reference value that for Type 1 Seyferts.
} 
and $\mathrm{F}_{\mathrm{X}}(2-10 \mathrm{keV}) / \mathrm{F}([\mathrm{OIII}])=1.1 \pm 0.8$, respectively.

Fig. 8 shows the hard $\mathrm{X}$-ray $(2-10 \mathrm{keV})$ versus the [OIII] emission line luminosities before (Left) and after (Right) the Compton-thickness correction. For LINERs, the former quantities are related by (circles in Fig. 8):

$$
\log L_{X}(2-10 k e V)=(10 \pm 2)+(0.8 \pm 0.1) \log L([O I I I])
$$

Note that Compton-thin and Compton-thick LINERs populate the same location than Compton-thin and Compton-thick Seyferts, respectively. Moreover, unobscured QSOs nicely extend to the brightest regime of the correlation. Note that there is a lack of high [OIII] luminosities seen in Compton-thin LINERs compared with Compton-thick LINERs. This is due to a selection effect since we are selecting LLAGN.

After Compton-thick corrections all the families show a linear correlation:

$$
\log L_{X}(2-10 k e V)=(3.2 \pm 1.4)+(0.95 \pm 0.03) \log L([O I I I])
$$

This correlation agrees with that recently found by Lamastra et al. (2009) for a sample of Compton-thin type 2 Seyferts. The good correlation of all the families of AGN, including LINERs (correlation coefficient of $\mathrm{r}=0.92$ ), is an argument favoring the same emission mechanism for all the objects.

Dudik et al. (2005) have found an Eddington ratio $L_{\text {bol }} / L_{E d d}=7 \times 10^{-6}$ for a sample of LINERs. These low accretion rates would indicate an inefficient accretion flow $\left(<10^{-3}\right.$ defined in Terashima et al. 2002). The Eddington ratio is defined as $L_{\text {bol }} / L_{\text {Edd }}$. Since the bolometric luminosity, $L_{\text {bol }}$, estimated as in Dudik et al. (2005), is directly related to the hard X-ray luminosity as $\mathrm{L}_{\text {bol }}=34 \times \mathrm{L}(2-10 \mathrm{keV})$, Compton-thickness correction may have an impact in the derived values of the Eddington ratios and hence in their interpretation.

We have revisited this question in LINERs by calculating their Eddington ratios with the Compton-thick corrected luminosities. Considering $\mathrm{L}_{\mathrm{Edd}}=1.26 \times 10^{38} \mathrm{M}_{\mathrm{BH}} / \mathrm{M}_{\odot} \mathrm{erg} \mathrm{s}^{-1}$ (Peterson 1997), we have estimated the black-hole masses by using the correlation between stellar velocity dispersion and black hole mass reported by Tremaine et al. (2002):

$$
\log \left(M_{B H}(M \odot)\right)=8.13+4.02 \log \left(\sigma\left(k m s^{-1}\right) / 200\right)
$$


The velocity dispersions have been taken from Hyperledd6, where they are available for 63 out of the 82 objects (Table 1, Cols. 10 and 11). The distribution of black hole masses is plotted in Fig. 9 (Dashed from up-left to down-right region). They range from $\log \left(\mathrm{M}_{\mathrm{BH}}\right)=6$ to $\log \left(M_{B H}\right)=9.5$ with a median value of $\log \left(M_{B H}\right)=8.22 \pm$ (and standard deviation of 0.65). Our values agree with those for the nine objects in common with Walsh et al. (2008), with STIS multi-spec data. Dudik et al. (2005) derived the black hole masses following Ferrarese et al. (2001) instead of the above equation. For comparison purposes we have also estimated the black-hole masses using this equation (Fig. 9, dashed from down-left to up-right region). Using Ferrarese et al. (2001) we derive a median value of $\log \left(\mathrm{M}_{\mathrm{BH}}\right)=$ $8.16 \pm$ (and standard deviation of 0.61), compatible with the values reported by using the approximation by Tremaine et al. (2002).

Fig. 10 shows L(2-10 keV) versus the black hole mass, before (Left) and after (Right) Compton-thickness correction. The data reported for type 1 and 2 Seyferts by Panessa et al. (2006) have been included. LINER black hole masses tend to be larger than those for type 2 Seyferts reported by Panessa et al. (2006) (average $\left.\log \left(\mathrm{M}_{\mathrm{BH}}\right)=7.0\right)$. Panessa et al. (2006) took their black hole masses from the literature, from kinematics methods to reverberation mapping or from the mass-velocity dispersion correlations. However, we do not expect any noticeable difference coming from this assumptions because our LINER black hole mass estimations are consistent with other methods (See Fig. 9). We have verified that the difference between black hole masses of Seyferts and LINERs can be attributed to the different distribution in morphological types, LINERs being hosted by earlier morphological type galaxies.

Before the Compton-thickness correction the median Eddington ratio was $\mathrm{L}_{\text {bol }} / \mathrm{L}_{\text {Edd }}=$ $3.2 \times 10^{-6}\left(\mathrm{~L}_{\text {bol }} / \mathrm{L}_{\text {Edd }}=2.8 \times 10^{-6}\right.$, assuming the value of $\mathrm{Lbol}=30 \times \mathrm{L}(2-10 \mathrm{keV})$, taken from Cappi et al. 2006), consistent with the result by Dudik et al. (2005). Nevertheless, after the Compton-thickness correction, this ratio increases up to $\mathrm{L}_{\text {bol }} / \mathrm{L}_{\text {Edd }}=1.9 \times 10^{-5}\left(\mathrm{~L}_{\text {bol }} / \mathrm{L}_{\text {Edd }}=\right.$ $1.8 \times 10^{-5}$, assuming Lbol $\left.=30 \times \mathrm{L}(2-10 \mathrm{keV})\right)$. Type 2 Seyfert galaxies by Cappi et al. (2006) (also Panessa et al. 2006) cover the same range of Eddington ratios, although they tend to be located close to $L_{\text {bol }} / L_{\text {Edd }} \simeq 1 \times 10^{-3}$. Type 1 Seyferts tend to be located at higher Eddington ratios. LINERs tend to be located closer to $L_{\text {bol }} / L_{\text {Edd }} \simeq 1 \times 10^{-5}$. The fact that Seyferts appear in later morphological types, typically Sab against S0 in LINERs, could explain the observed trend.

Radiatively inefficient accretion is invoked to explain such low Eddington ratios. Merloni et al. (2003) argued that the accretion mode changes to radiatively inneficient process below $10^{-3}$. Following it, all but seven sources are in the regime of radiatively inneficient process. There-

\footnotetext{
${ }^{6}$ http://leda.univ-lyon1.fr/
} 
fore, for the overall picture of LINERs, their $L_{\text {bol }} / L_{\text {Edd }}$ ratio, as well as for the type 2 Seyferts reported by Panessa et al. (2006), is within the inefficient regime. Therefore, a combination of obscuration and inefficient accretion is needed to explain their properties. LINERs, therefore, share the range of accretion rates and obscuration of type 2 Seyferts, but occupying lower accretion rates and higher obscurations than their parent population of Seyferts.

\section{Obscuration and SED}

Ho et al. (1999) (see Ho 2008 for a review) showed that the SED of LLAGN with $\mathrm{L}_{\text {bol }} / \mathrm{L}_{\text {Edd }}<1 \times 10^{-3}$ emphasizing that the big blue bump seen in luminous AGN is absent in these sources, shifting the peak to the mid IR frequencies. Two possibilities exist: (1) Their nuclei are obscured and their UV photons are re-emit toward mid-IR frequencies or (2) they have a inherent different SED.

Ho (2008) ruled out the obscuration possibility mainly because of the lack of obscuration seen at X-ray in LINERs. However, in this study (also GM+09) we have demonstrated that they show X-ray obscuration (similar distribution of column densities than type 2 Seyferts) and a high percentage of them could be highly obscured AGN (higher percentage than type 2 Seyferts in the Compton-thick regime). Therefore, the unobstructed view of the nucleus discussed by Ho (2008) is not so clear and obscuration of their nuclei becomes again relevant.

In the case of a intrinsically different SED, this could produce a lack of UV photons and, thus, a faint production of the [OIII] emission. Therefore, this effect could affect those objects with large $F_{X}(2-10 \mathrm{keV}) / F([\mathrm{OIII}])$. Thirty seven objects showed a high ratio $\left(\log \left(\mathrm{F}_{\mathbf{X}}(2-10 \mathrm{keV}) / \mathrm{F}([\mathrm{OIII}])\right)>0.5\right)$. However, it is worth noticing that the same effect can be produced by a wrong estimation of the extinction (discussed in Section 2.2). Eleven out of these 37 objects were not corrected from extinction, assuming a minimum of $\mathrm{H} \alpha / \mathrm{H} \beta=3.1$. Excluding them, 26 objects showed $\log \left(\mathrm{F}_{\mathrm{X}}(2-10 \mathrm{keV}) / \mathrm{F}([\mathrm{OIII}])\right)>0.5$. However, a hydrogen column density higher than $\mathrm{NH}>10^{21} \mathrm{~cm}^{-2}$ is present in all but 5 cases (NGC 3945, NGC 4636, NGC 4696, IRAS 14348-1447 and MRK 848). In these five cases an intrinsic different SED might be the only explanation for their large $F_{X}(2-10 \mathrm{keV}) / F([\mathrm{OIII}])$. However, this is not the generality of the sample, in which the obscuration is a very important ingredient.

In fact, Maoz (2007) reported the X-ray-to-UV ratio $\left(\alpha_{\text {ox }}\right)$ for a sample of 13 LINERs. He found a ratio between $-1.4<\alpha_{\mathrm{ox}}<-0.8$. Seven out of the 13 objects are included in our sample. All of them show ratios $\mathrm{F}_{\mathrm{X}}(2-10 \mathrm{keV}) / \mathrm{F}([\mathrm{OIII}])>0.5$, as expected if these objects could show a deficit of UV photons. However, their NH column densities are higher than 
$\mathrm{NH}>10^{21} \mathrm{~cm}^{-2}$, that could also affect the UV band. Intriguingly, in spite of the lack of statistic (only seven objects), we have found a hint of correlation between $\alpha_{\text {ox }}$ and the NH column density, obtaining a coefficient of a correlation of $r=0.56$. NGC 5494 is clearly out of the correlation (excluding it, we obtain $\mathrm{r}=0.80$ ). A bigger sample of LINERs with hydrogen column densities as well as X-ray and UV luminosities would be needed before any strong conclusions can be made.

For the whole energy distribution, Nemmen et al. (2006) studied the LINER NGC 1097 SED, successfully reproducing using a model of an optically thin geometrically thick RIAF for the inner radii and an optically thick, geometrically thin disk for larger radii. They needed to add a contribution of young and obscured starburst to account for the UV excess emission. However, although this is the case for NGC 1097, we know that young circumnuclear starburst are not an important ingredient in most of LINERs (see GM+09). We need to study the LINER SED in a larger sample of objects to check if they can be explained with an ADAF model and the obscuration reported here.

\section{Summary and conclusions}

In this paper, we study the obscuration in LINER nuclei. We use X-ray spectral parameters, FeK $\alpha$ emission line and luminosities reported in $\mathrm{GM}+09$ and in this paper. We also make use of their optical properties traced by HST morphology, [OIII] emission line fluxes, optical extinction and environmental information.

Our key finding is that around $50 \%$ of our LINER sample shows Compton-thick signatures, according to the accessible diagnostics. This fraction is larger than that reported for Seyfert nuclei. Moreover, this high percentage of LINERs showing high obscuration consistent with the observed decrease of obscured AGN fraction with luminosity (Della Ceca et al. 2008).

This population of Compton-thick LINERs shows lower EW(FeK $\alpha)$. Brightman and Nandra (2008) suggest that the scattered emission dominating below $\sim 5 \mathrm{keV}$, what leads to a misinterpretation of the spectrum as an unobscured object while a nuclear harder component is present. As pointed by Brightman and Nandra (2008), the high contribution of the scattering component might suggest a low covering fraction of the torus or a high density of the gas. The thermal emission in LINER nuclei, however, seems to be another important ingredient since it is needed in the majority of LINERs, accounting for a high fraction of the emission above $\sim 2 \mathrm{keV}$. X-ray spectroscopic measurements above $10 \mathrm{keV}$ together with high sensitivity spectra around $6.4 \mathrm{keV}$ are required for a better understanding of their 
nature.

We have also investigated the connection between hydrogen column densities and the optical extinction and environment. The soft column density (NH1) is related with the diffuse emission around the AGN at least in some cases, and it correlates with the optical extinction. The hard column density (NH2) is much higher than the optical extinction, that might be associated to the inner parts of the AGN, buried at optical wavelengths. No relation was found between optical dust lanes and X-ray obscuration or Compton-thick LINERs. Finally, LINER nuclei show lower Eddington ratios than type 2 Seyferts, although they cover the same range of values. We want to remark that the Compton-thickness luminosity correction is very important for a proper estimation of their Eddington ratios.

Therefore, obscuration plays an important role in LINERs. Close to our findings, Dudik et al. (2009) found that a high extinction even at mid-IR frequencies is needed to explain the deficit of $\mathrm{NeV} \lambda \lambda 14 \mu \mathrm{m}$ compared with $\mathrm{NeV} \lambda \lambda 24 \mu \mathrm{m}$. In fact, their seven objects in common with our sample (NGC 1052, NGC 3507, NGC 4736, UGC05101, UGC 08696, NGC 6240 and NGC 7130) with deficit of NeV $\lambda \lambda 14 \mu \mathrm{m}$ show a high NH column density or have been classified as Compton-thick sources. High obscuration of the central source might also explain the lack of the UV bump in LINERs.

Our results seem to be consistent with an scenario in which LINER nuclei are characterized by two phases of obscuration. The hard X-ray obscuring material will be similar to that obtained for type 2 Seyferts and will be much more obscured than that expected to produce the optical extinction. A possibility that needs to be investigated is whether it is related to the putative dusty torus invoked by the unified model for AGN nuclei. Nenkova et al. (2002) proposed that the obscuring region in AGN is a toroidal distribution of dusty clouds. Ibar and Lira (2007) predicted an intrinsic Compton-thick source fraction of $58 \%$ for the clumpy torus scenario while the 'classical' torus produces a fraction of Compton-thick sources of $27 \%$. Our results are closer to the expectations for a clumpy torus. Outside the hard X-ray absorbing material, LINERs might also show a second phase of obscuration. This second

phase will be responsible for the optical extinction, and will completely bury the intrinsic continuum at optical wavelengths.

\section{Acknowledgements}

We acknowledge the referee for his/her helpful comments. We also thank D. Dultzin for fruitful discussions. We gratefully acknowledge F. Durret, J. Acosta, F. Carrera and E. 
Florido, members of OGM's PhD jury. We also thank to A. de Ugarte Postigo for the help to improve the text. This work was financed by DGICyT grants AYA 2003-00128, AYA 2006-01325, AYA 2007-62190 and the Junta de Andalucía TIC114. OGM acknowledges financial support from the Ministerio de Educación y Ciencia through the Spanish grant FPI BES-2004-5044 and research fellowship of STFC. This research has made use of data obtained from the Chandra Data Archive provided by the Chandra X-ray Center (CXC) $X M M$-Newton Data Archive provided by the XMM-Newton Science Archive (XSA). This paper is also partially based on NASA/ESA Hubble Space Telescope observations. This research made use of the NASA/IPAC extragalactic database (NED), which is operated

by the Jet Propulsion Laboratory under contract with the National Aeronautics and Space Administration, and Hyperleda database.

\section{REFERENCES}

Antonucci, R.: 1993, Annual Review of Astronomy and Astrophysics 31, 473

Ballo, L., Braito, V., Ceca, R. D., Maraschi, L., Tavecchio, F., and Dadina, M.: 2004, Astrophysical Journal 600, 634

Barnes, J. E. and Hernquist, L.: 1996, Astrophysical Journal 471, 115

Bassani, L., Dadina, M., Maiolino, R., Salvati, M., Risaliti, G., della Ceca, R., Matt, G., and Zamorani, G.: 1999, Astrophysical Journal Supplement Series 121, 473

Bassani, L., Dadina, M., Maiolino, R., Salvati, M., Risaliti, G., della Ceca, R., Matt, G., and Zamorani, G.: 2000, VizieR Online Data Catalog 212, 10473

Beckmann, V., Soldi, S., Shrader, C. R., Gehrels, N., and Produit, N.: 2006, ApJ 652, 126

Bianchi, S., Matt, G., Balestra, I., Guainazzi, M., and Perola, G. C.: 2004, Astronomy and Astrophysics 422, 65

Boller, T., Keil, R., Hasinger, G., Costantini, E., Fujimoto, R., Anabuki, N., Lehmann, I., and Gallo, L.: 2003, Astronomy and Astrophysics 411, 63

Brightman, M. and Nandra, K.: 2008, MNRAS 390, 1241

Cappi, M., Panessa, F., Bassani, L., Dadina, M., Dicocco, G., Comastri, A., della Ceca, R., Filippenko, A. V., Gianotti, F., Ho, L. C., Malaguti, G., Mulchaey, J. S., Palumbo, G. G. C., Piconcelli, E., Sargent, W. L. W., Stephen, J., Trifoglio, M., and Weaver, K. A.: 2006, Astronomy and Astrophysics 446, 459 
Carrillo, R., Masegosa, J., Dultzin-Hacyan, D., and Ordonez, R.: 1999, Revista Mexicana de Astronomia y Astrofisica 35, 187

Ceca, R. D., Ballo, L., Tavecchio, F., Maraschi, L., Petrucci, P. O., Bassani, L., Cappi, M., Dadina, M., Franceschini, A., Malaguti, G., Palumbo, G. G. C., and Persic, M.: 2002, Astrophysical Journal 581, L9

Dadina, M.: 2008, A\&A 485, 417

Della Ceca, R., Caccianiga, A., Severgnini, P., Maccacaro, T., Brunner, H., Carrera, F. J., Cocchia, F., Mateos, S., Page, M. J., and Tedds, J. A.: 2008, A\&A 487, 119

Dewangan, G. C. and Griffiths, R. E.: 2005, ApJL 625, L31

Downes, D. and Solomon, P. M.: 1998, ApJ 507, 615

Duc, P.-A., Mirabel, I. F., and Maza, J.: 1997, Astronomy and Astrophysics Supplement Series 124, 533

Dudik, R. P., Satyapal, S., Gliozzi, M., and Sambruna, R. M.: 2005, Astrophysical Journal 620, 113

Dudik, R. P., Satyapal, S., and Marcu, D.: 2009, ApJ 691, 1501

Evans, A. S., Mazzarella, J. M., Surace, J. A., and Sanders, D. B.: 2002, ApJ 580, 749

Ferrarese, L., Pogge, R. W., Peterson, B. M., Merritt, D., Wandel, A., and Joseph, C. L.: 2001, ApJL 555, L79

Ghisellini, G., Haardt, F., and Matt, G.: 1994, Monthly Notices of the Royal Astronomical Society 267, 743

Gonzalez-Delgado, R. M., Fernandes, R. C., Perez, E., Martins, L. P., Storchi-Bergmann, T., Schmitt, H., Heckman, T., and Leitherer, C.: 2004, Astrophysical Journal 605, 127

Gonzalez-Delgado, R. M., Perez, E., Fernandes, R. C., and Schmitt, H.: 2008a, Astronomical Journal 135, 747

Gonzalez-Delgado, R. M., Perez, E., Fernandes, R. C., and Schmitt, H.: 2008b, Astronomical Journal 135, 747

Gonzalez-Martin, O., Masegosa, J., Marquez, I., Guainazzi, M., and Jimenez-Bailon, E.: 2009, ArXiv e-prints 
Gonzalez-Martin, O., Masegosa, J., Marquez, I., Guerrero, M. A., and Dultzin-Hacyan, D.: 2006, Astronomy and Astrophysics 460, 45

Gonzalez-Martin et al., O.: 2008, PhD Thesis, Univerity of Granada, URL: http://www.star.le.ac.uk/ gmo4/O.Gonzalez-Martin-part1.pdf

Greenawalt, B., Walterbos, R. A. M., and Braun, R.: 1997, Astrophysical Journal 483, 666

Guainazzi, M., Fiore, F., Matt, G., and Perola, G. C.: 2001, Monthly Notices of the Royal Astronomical Society 327, 323

Guainazzi, M., Matt, G., and Perola, G. C.: 2005, Astronomy and Astrophysics 444, 119

Ho, L. C.: 2008, ARA\&A 46, 475

Ho, L. C., Feigelson, E. D., Townsley, L. K., Sambruna, R. M., Garmire, G. P., Brandt, W. N., Filippenko, A. V., Griffiths, R. E., Ptak, A. F., and Sargent, W. L. W.: 2001, Astrophysical Journal 549, L51

Ho, L. C., Filippenko, A. V., Sargent, W. L. W., and Peng, C. Y.: 1997, Astrophysical Journal Supplement Series 112, 391

Ibar, E. and Lira, P.: 2007, Astronomy and Astrophysics 466, 531

Imanishi, M., Terashima, Y., Anabuki, N., and Nakagawa, T.: 2003, Astrophysical Journal 596, L167

Jimenez-Bailon, E., Piconcelli, E., Guainazzi, M., Schartel, N., Rodriguez-Pascual, P. M., and Santos-Lleo, M.: 2005, Astronomy and Astrophysics 435, 449

Keel, W. C.: 1983, Astrophysical Journal 269, 466

Keel, W. C., Kennicutt, R. C., Hummel, E., and van der Hulst, J. M.: 1985, Astronomical Journal 90, 708

Koski, A. T.: 1978, Astrophysical Journal 223, 56

Krongold, Y., Dultzin-Hacyan, D., and Marziani, P.: 2003, in V. Avila-Reese, C. Firmani, C. S. Frenk, and C. Allen (eds.), Revista Mexicana de Astronomia y Astrofisica Conference Series, Vol. 17 of Revista Mexicana de Astronomia y Astrofisica Conference Series, pp 105-105

Lamastra, A., Bianchi, S., Matt, G., Perola, G. C., Barcons, X., and Carrera, F. J.: 2009, ArXiv e-prints 
Leahy, D. A. and Creighton, J.: 1993, MNRAS 263, 314

Magdziarz, P. and Zdziarski, A. A.: 1995, MNRAS 273, 837

Maiolino, R.: 2001, X-ray Astronomy: Stellar Endpoints, AGN, and the Diffuse X-ray Background 599, 199

Maiolino, R., Comastri, A., Gilli, R., Nagar, N. M., Bianchi, S., Beker, T., Colbert, E., Krabbe, A., Marconi, A., Matt, G., and Salvati, M.: 2003, Monthly Notices of the Royal Astronomical Society 344, L59

Maiolino, R. and Rieke, G. H.: 1995, Astrophysical Journal 454, 95

Maiolino, R., Salvati, M., Bassani, L., Dadina, M., della Ceca, R., Matt, G., Risaliti, G., and Zamorani, G.: 1998, Astronomy and Astrophysics 338, 781

Maoz, D.: 2007, MNRAS 377, 1696

Marziani, P., Sulentic, J. W., Zamanov, R., Calvani, M., Dultzin-Hacyan, D., Bachev, R., and Zwitter, T.: 2003, ApJS 145, 199

Matt, G.: 1997, Memorie della Societa Astronomica Italiana 68, 127

Merloni, A., Heinz, S., and di Matteo, T.: 2003, MNRAS 345, 1057

Mihos, J. C. and Hernquist, L.: 1994, ApJL 437, L47

Montero-Dorta, A. D., Croton, D. J., Yan, R., Cooper, M. C., Newman, J. A., Georgakakis, A., Prada, F., Davis, M., Nandra, K., and Coil, A.: 2008, MNRAS pp 1355-+

Moustakas, J. and Kennicutt, R. C.: 2006, Astrophysical Journal 651, 155

Mulchaey, J. S., Koratkar, A., Ward, M. J., Wilson, A. S., Whittle, M., Antonucci, R. R. J., Kinney, A. L., and Hurt, T.: 1994, ApJ 436, 586

Nagar, N. M., Falcke, H., and Wilson, A. S.: 2005, Astronomy and Astrophysics 435, 521

Nandra, K. and Iwasawa, K.: 2007, MNRAS 382, L1

Nandra, K., O'Neill, P. M., George, I. M., and Reeves, J. N.: 2007, Monthly Notices of the Royal Astronomical Society 382, 194

Nemmen, R. S., Storchi-Bergmann, T., Yuan, F., Eracleous, M., Terashima, Y., and Wilson, A. S.: 2006, ApJ 643, 652 
Nenkova, M., Ivezic, Z., and Elitzur, M.: 2002, Astrophysical Journal 570, L9

Osterbrock, D. E. and Ferland, G. J.: 2006, Astrophysics of gaseous nebulae and active galactic nuclei

Panessa, F. and Bassani, L.: 2002, Astronomy and Astrophysics 394, 435

Panessa, F., Bassani, L., Cappi, M., Dadina, M., Barcons, X., Carrera, F. J., Ho, L. C., and Iwasawa, K.: 2006, Astronomy and Astrophysics 455, 173

Panessa, F., Bassani, L., de Rosa, A., Bird, A. J., Dean, A. J., Fiocchi, M., Malizia, A., Molina, M., Ubertini, P., and Walter, R.: 2008, A\&A 483, 151

Panessa, F., Wolter, A., Pellegrini, S., Fruscione, A., Bassani, L., Ceca, R. D., Palumbo, G. G. C., and Trinchieri, G.: 2005, Astrophysical Journal 631, 707

Perola, G. C., Matt, G., Cappi, M., Fiore, F., Guainazzi, M., Maraschi, L., Petrucci, P. O., and Piro, L.: 2002, Astronomy and Astrophysics 389, 802

Peterson, B. M.: 1997, An Introduction to Active Galactic Nuclei, An introduction to active galactic nuclei, Publisher: Cambridge, New York Cambridge University Press, 1997 Physical description xvi, 238 p. ISBN 0521473489

Piconcelli, E., Jimenez-Bailón, E., Guainazzi, M., Schartel, N., Rodríguez-Pascual, P. M., and Santos-Lleó, M.: 2005, A\&A 432, 15

Ptak, A., Heckman, T., Levenson, N. A., Weaver, K., and Strickland, D.: 2003, Astrophysical Journal 592, 782

Rees, M. J.: 1984, Annual Review of Astronomy and Astrophysics 22, 471

Risaliti, G.: 2002, A\&A 386, 379

Risaliti, G. and Elvis, M.: 2004, Vol. 308, p. 187

Risaliti, G., Maiolino, R., and Salvati, M.: 1999, Astrophysical Journal 522, 157

Rovilos, E., Georgantopoulos, I., Tzanavaris, P., Pracy, M., Whiting, M., Woods, D., and Goudis, C.: 2009, ArXiv e-prints

Rupke, D. S. N., Veilleux, S., and Baker, A. J.: 2008, ApJ 674, 172

Sarzi, M., Rix, H.-W., Shields, J. C., Ho, L. C., Barth, A. J., Rudnick, G., Filippenko, A. V., and Sargent, W. L. W.: 2005, ApJ 628, 169 
Teng, S. H., Veilleux, S., Anabuki, N., Dermer, C. D., Gallo, L. C., Nakagawa, T., Reynolds, C. S., Sanders, D. B., Terashima, Y., and Wilson, A. S.: 2008, ArXiv e-prints

Terashima, Y., Iyomoto, N., Ho, L. C., and Ptak, A. F.: 2002, Astrophysical Journal Supplement Series 139, 1

Tremaine, S., Gebhardt, K., Bender, R., Bower, G., Dressler, A., Faber, S. M., Filippenko, A. V., Green, R., Grillmair, C., Ho, L. C., Kormendy, J., Lauer, T. R., Magorrian, J., Pinkney, J., and Richstone, D.: 2002, Astrophysical Journal 574, 740

Turner, T. J., George, I. M., Nandra, K., and Mushotzky, R. F.: 1998, Astrophysical Journal 493, 91

Veilleux, S., Kim, D.-C., Sanders, D. B., Mazzarella, J. M., and Soifer, B. T.: 1995, Astrophysical Journal Supplement Series 98, 171

Veilleux, S. and Osterbrock, D. E.: 1987, ApJS 63, 295

Veilleux, S., Sanders, D. B., and Kim, D.-C.: 1999, Astrophysical Journal 522, 139

Vignali, C., Comastri, A., Cappi, M., Palumbo, G. G. C., Matsuoka, M., and Kubo, H.: 1999, Astrophysical Journal 516, 582

Walsh, J. L., Barth, A. J., Ho, L. C., Filippenko, A. V., Rix, H.-W., Shields, J. C., Sarzi, M., and Sargent, W. L. W.: 2008, AJ 136, 1677

Winter, L. M., Mushotzky, R. F., Reynolds, C. S., and Tueller, J.: 2009, ApJ 690, 1322

York, D. G., Khare, P., Vanden Berk, D., Kulkarni, V. P., Crotts, A. P. S., Lauroesch, J. T., Richards, G. T., Schneider, D. P., Welty, D. E., Alsayyad, Y., Kumar, A., Lundgren, B., Shanidze, N., Smith, T., Vanlandingham, J., Baugher, B., Hall, P. B., Jenkins, E. B., Menard, B., Rao, S., Tumlinson, J., Turnshek, D., Yip, C.-W., and Brinkmann, J.: 2006, MNRAS 367, 945

Zhang, W. M., Soria, R., Zhang, S. N., Swartz, D. A., and Liu, J. F.: 2009, ApJ 699, 281 


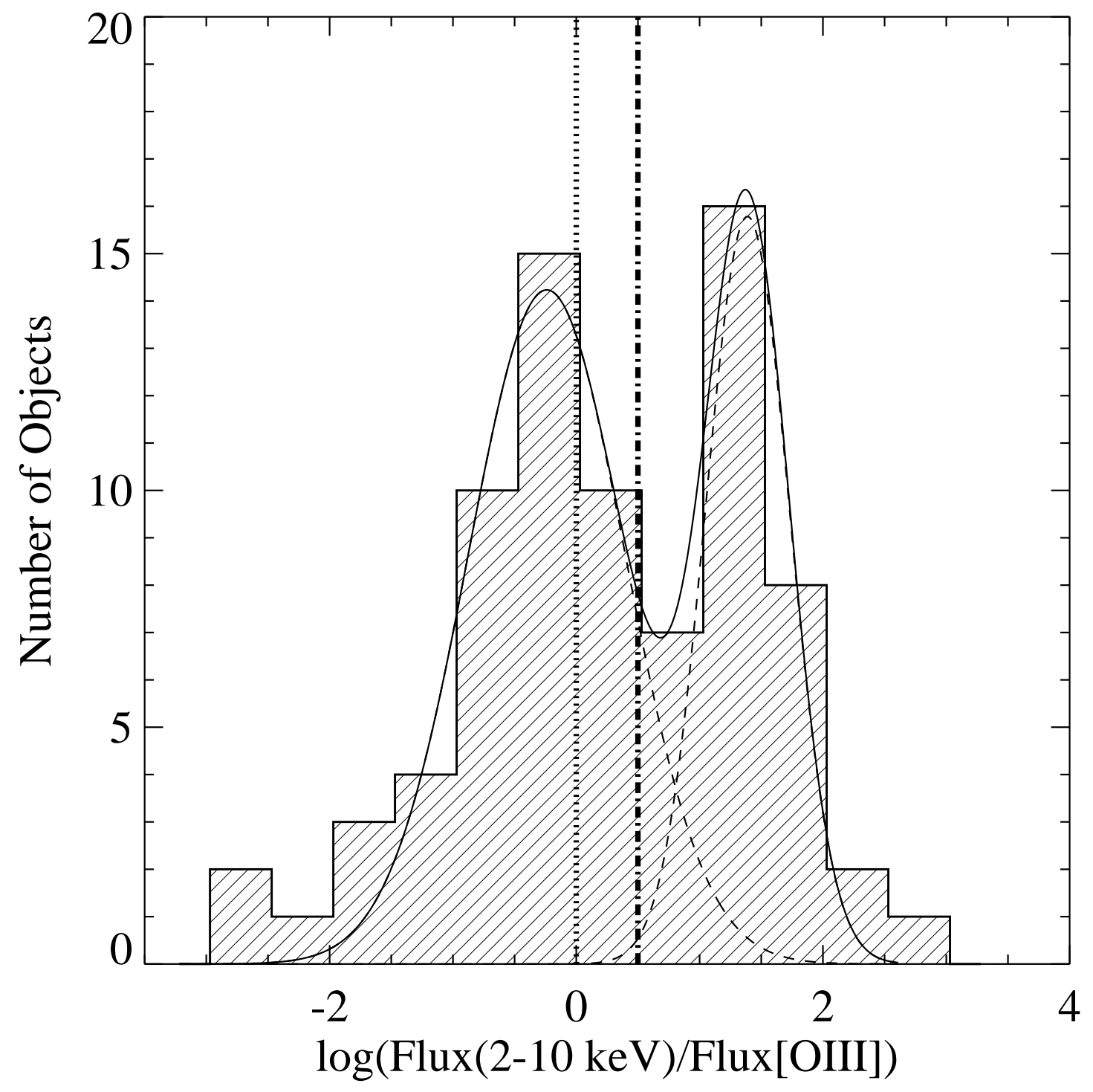

Fig. 1.- Histogram for the ratio between X-ray 2-10 keV energy band fluxes and reddeningcorrected [OIII] emission line fluxes. The dot-dashed line correspond to the limit found by Maiolino et al. (1998) between Compton-thin and Compton-thick sources. The dotted line correspond to the conservative limit adopted by Cappi et al. (2006). Continuous line shows the best fit to a two Gaussians model and the dashed lines show the Gaussian components. 


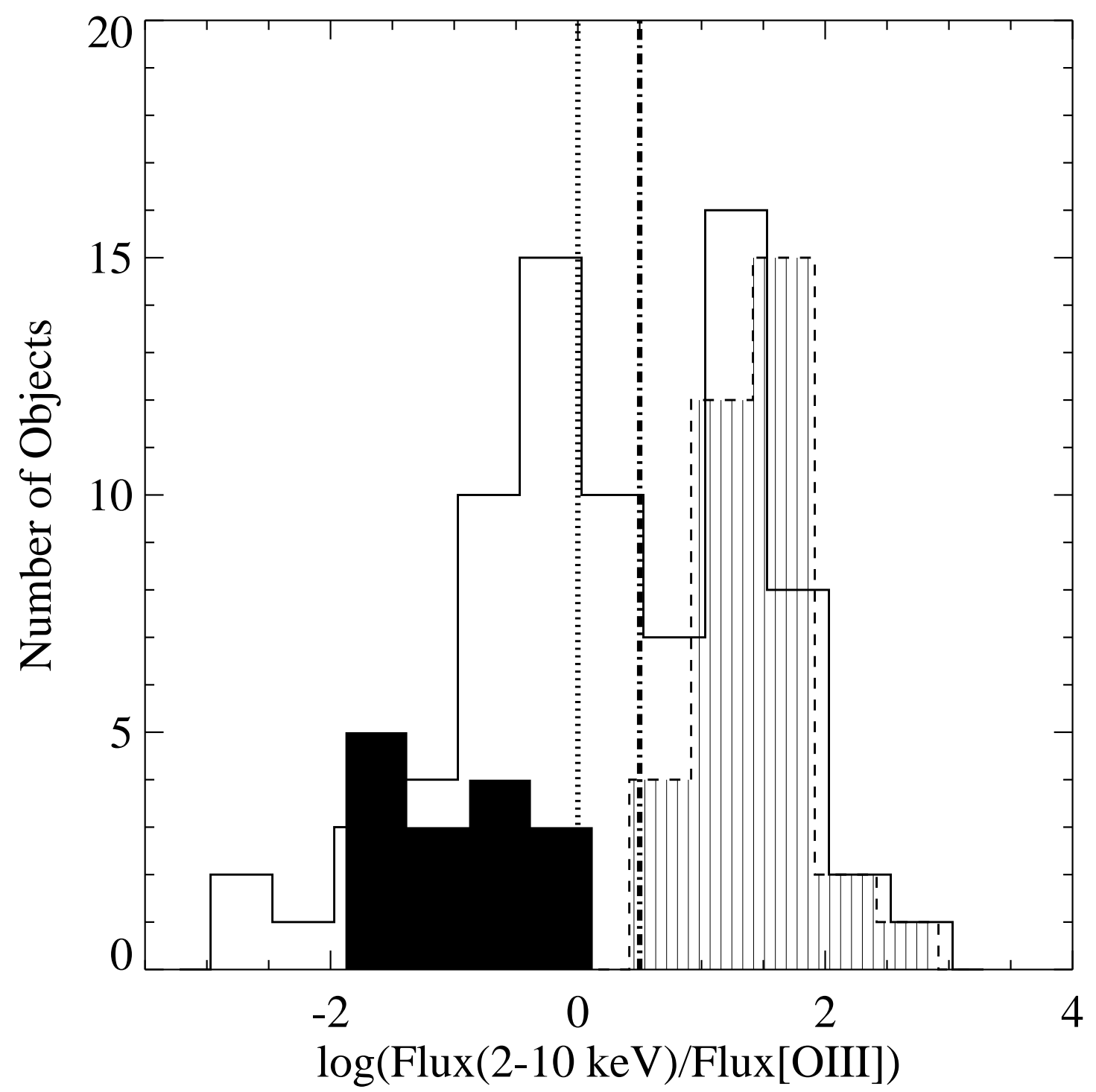

Fig. 2.- Histogram for the ratio between X-ray 2-10 keV energy band fluxes and reddeningcorrected [OIII] emission line fluxes. The dot-dashed line correspond to the limit found by Maiolino et al. (1998) between Compton-thin and Compton-thick sources. The dotted line correspond to the conservative limit adopted by Cappi et al. (2006). The empty histogram is the distribution obtained with our LINER sample. The black-filled histogram contains Compton-thick sources by Bassani et al. (1999) and the dashed filled histogram shows the unobscured PG QSOs reported by Jimenez-Bailon et al. (2005) and Piconcelli et al. (2005). 


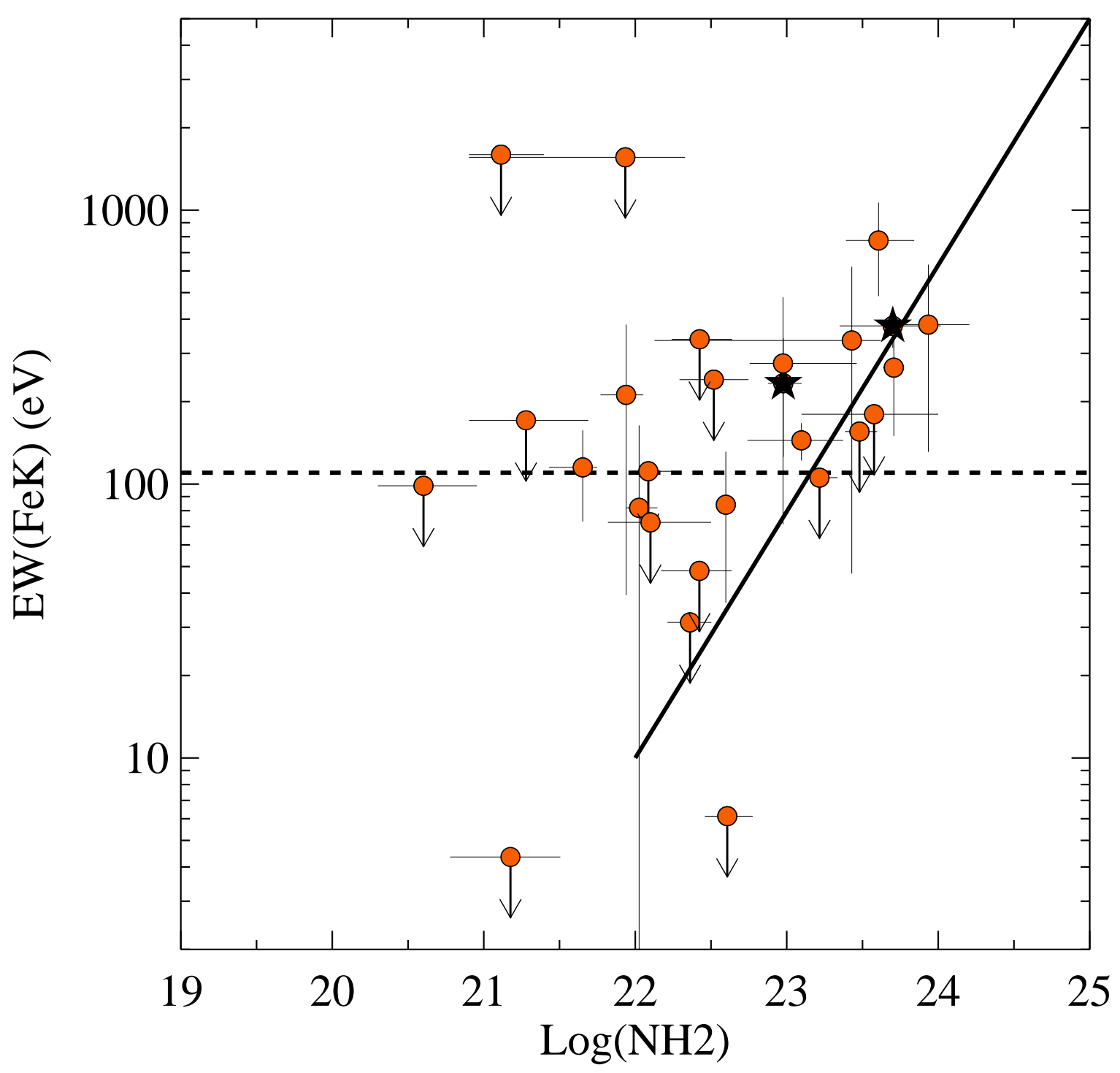

Fig. 3.- EW of the FeK $\alpha$ emission line versus the NH2 column density. Black stars are confirmed Compton-thick (see Sect. 4.1). The horizontal dotted line is the mean level reported by Guainazzi et al. (2005) for a sample of type 2 Seyferts. The diagonal continuous line shows the predicted line reported by Ghisellini et al. (1994) for attenuation by photoabsorption and Compton scattering. 


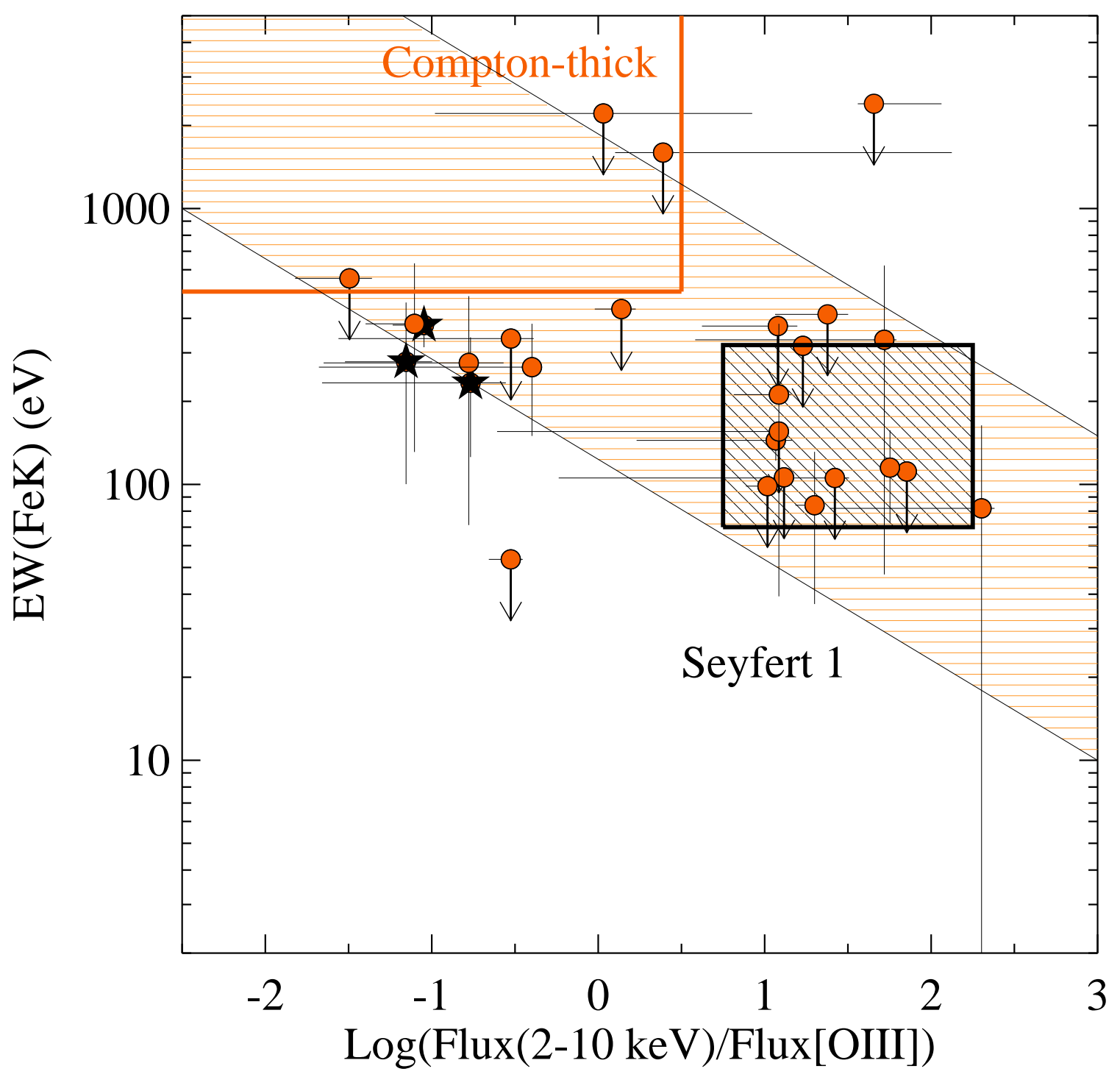

Fig. 4.- EW of the FeK $\alpha$ line versus the ratio between the observed 2-10 keV flux and the dereddened [OIII] emission line flux. Black stars are Compton-thick sources. The region filled with red horizontal lines shows the trend between this two quantities shown by Bassani et al. (2000). The square filled with diagonal black lines shows type 1 Seyferts location and the red-continuous line shows Compton-thick sources location (Bassani et al. 2000). 


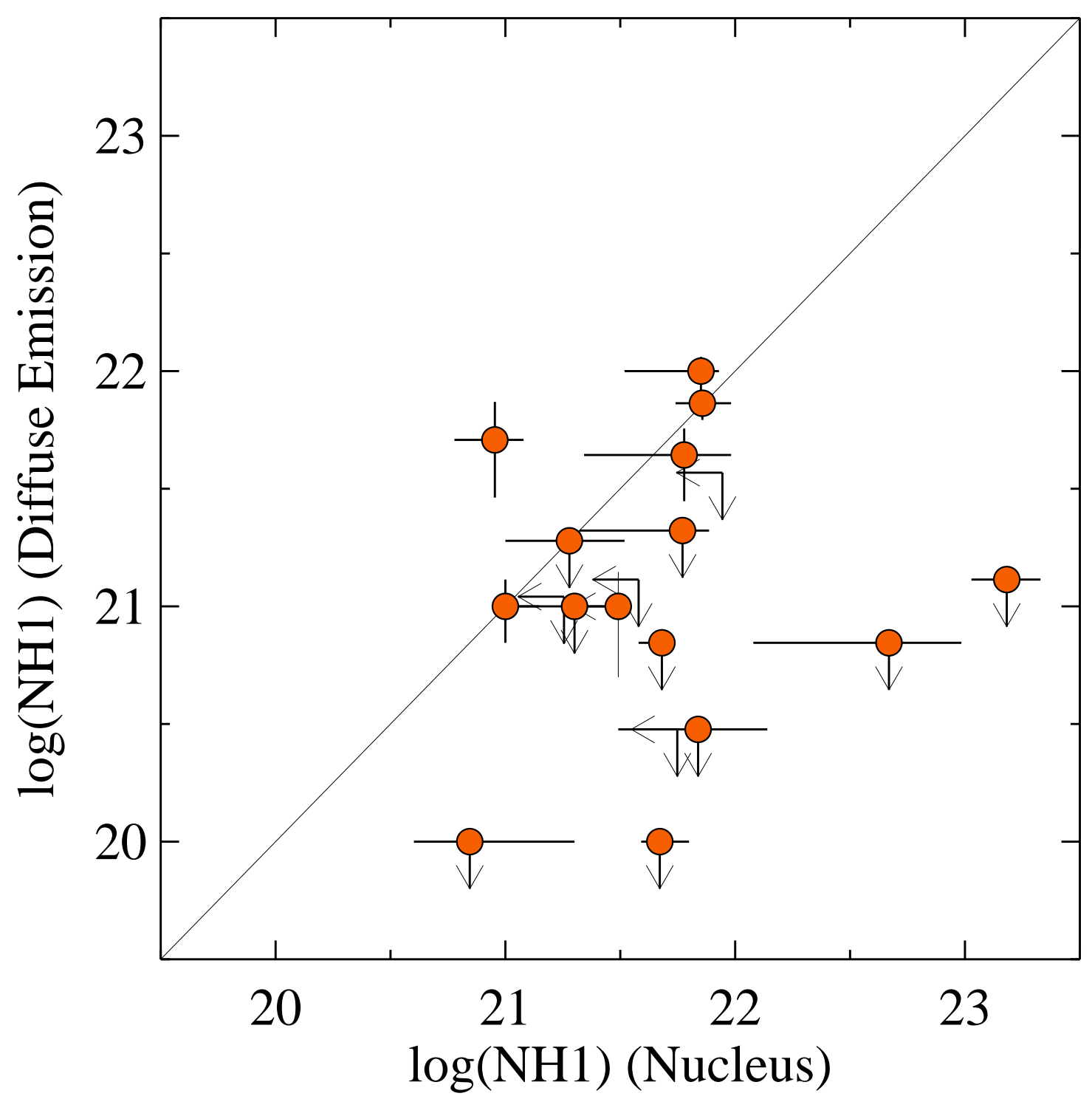

Fig. 5.- NH1 column density of the diffuse emission (NH1(Diffuse Emission)) versus the NH1 column density of the nuclear emission (NH1(Nucleus)). Arrows are upper limits. The unity slope is shown as continuous line. 

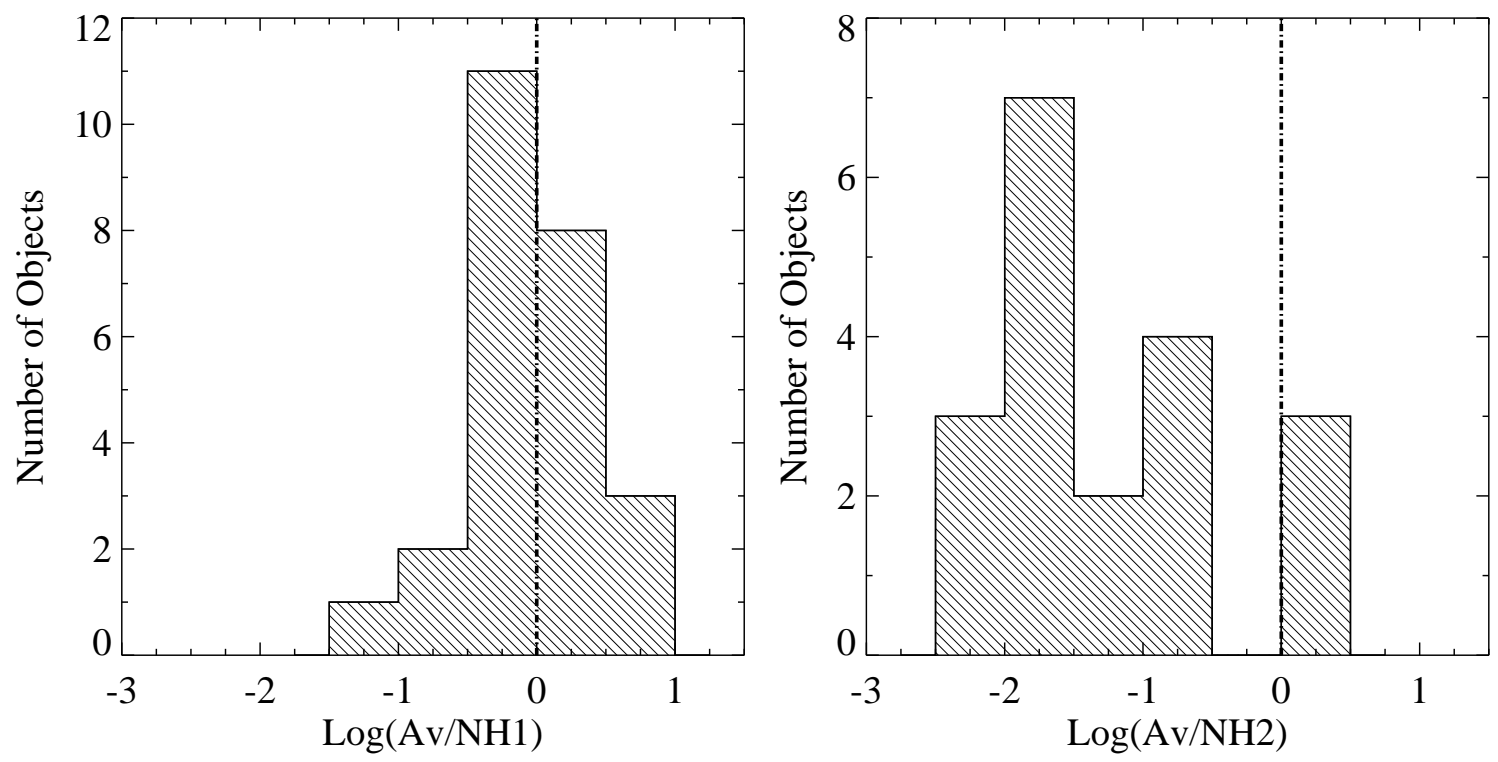

Fig. 6.- Histograms of the ratio between the NH1 column density and optical extinction $\mathrm{Av}$ (Left) and NH2 and Av (Right). The dot-dashed line is the expected locus when the optical extinction Av can explain the X-ray column density $(\mathrm{Av} / \mathrm{NH}=1)$. Optical extinction Av is converted into $\mathrm{cm}^{-2}$ units by assuming a Galactic ratio $A v / \mathrm{NH}=5 \times 10^{22} \mathrm{~cm}^{-2}$. 


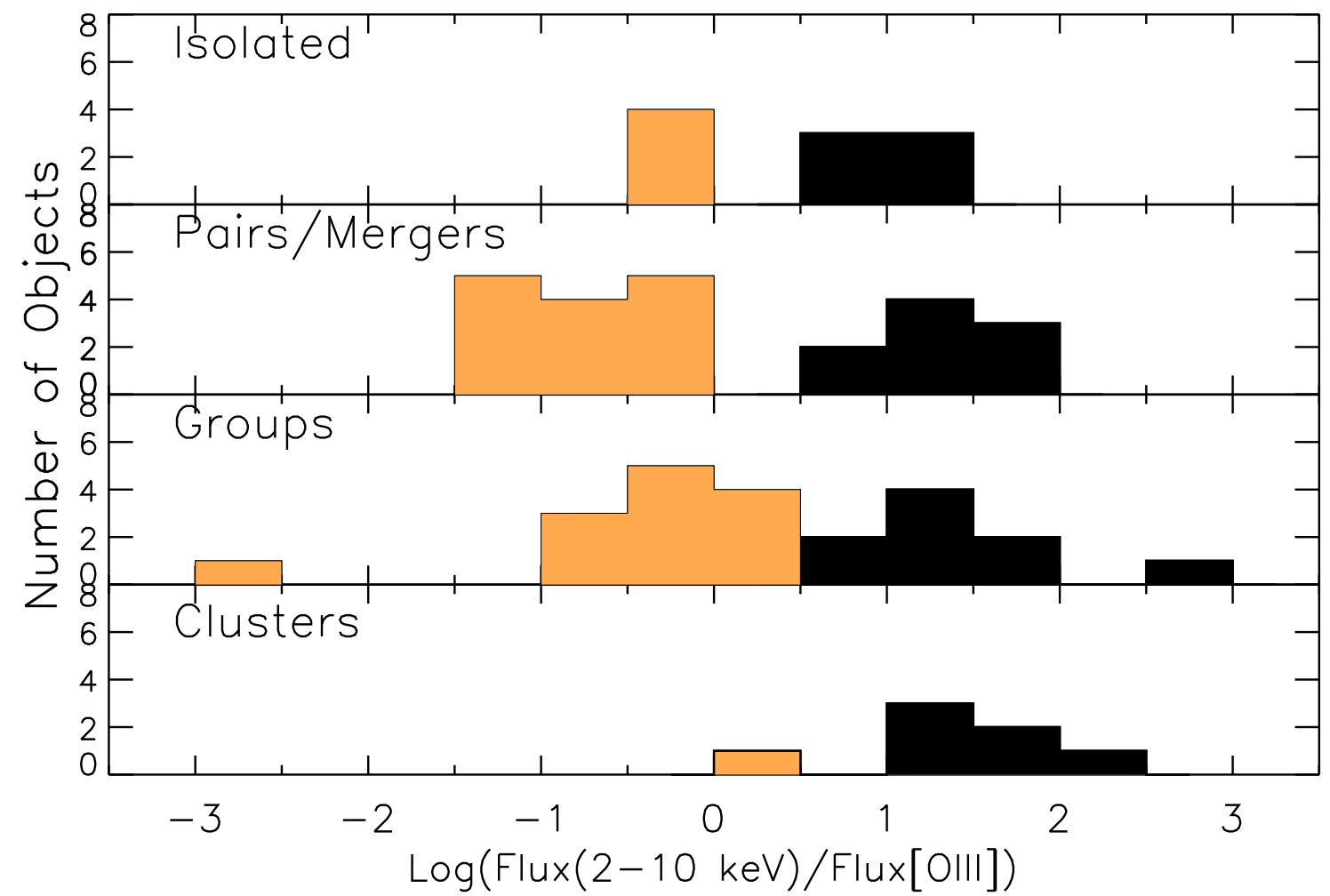

Fig. 7.- Histogram for the $\mathrm{L}(2-10 \mathrm{keV}) / \mathrm{L}([\mathrm{OIII}])$ for the different environments: isolated (a), pairs and mergers (b), groups (c) and clusters (d), from top to bottom. Red filled histograms are Compton-thick sources and black-filled histograms are Compton-thin sources (according to the $\mathrm{L}(2-10 \mathrm{keV}) / \mathrm{L}([\mathrm{OIII}])$ ratio). 


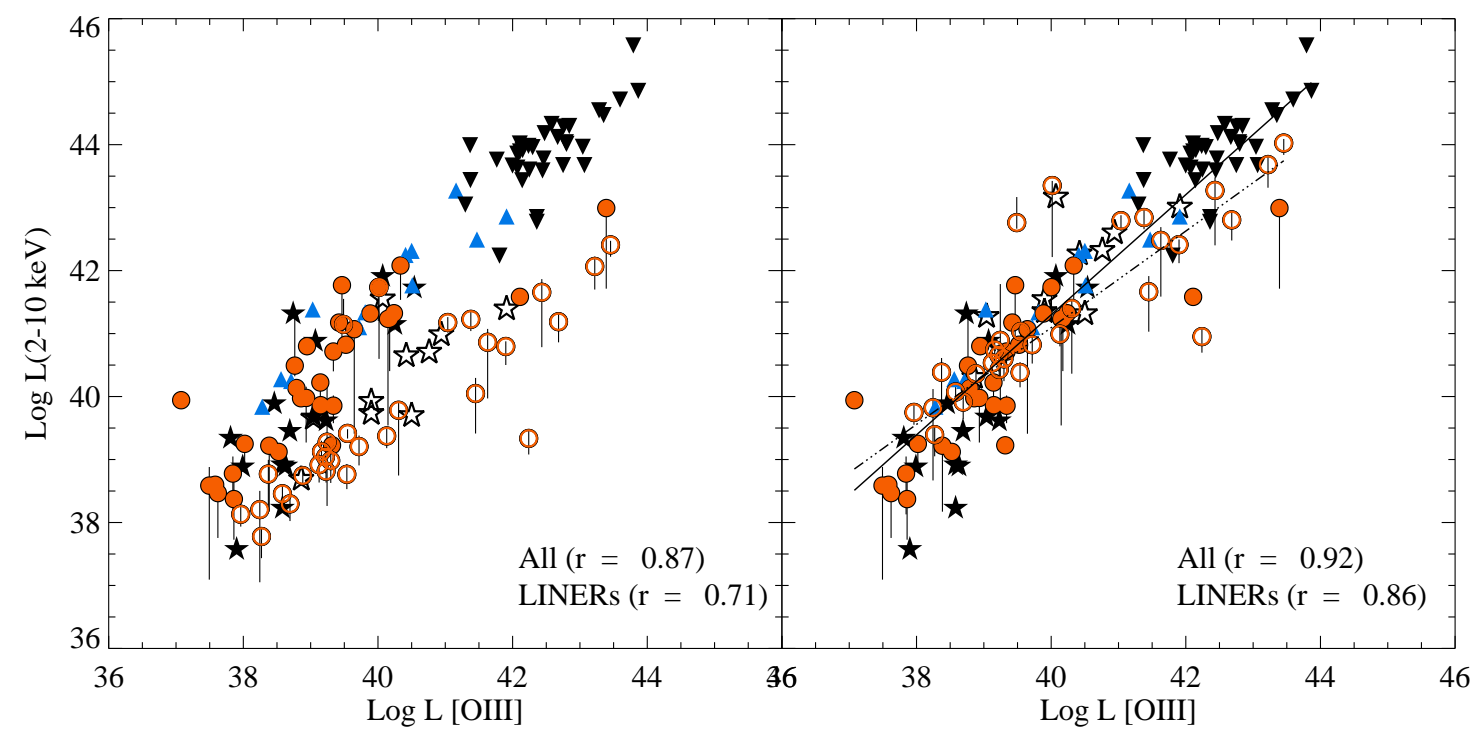

Fig. 8.- L $(2-10 \mathrm{keV})$ versus $\mathrm{L}([\mathrm{OIII}])$ before (left) and after (right) the Compton-thickness correction. Red-filled circles are Compton-thin LINERs, open circles correspond to Comptonthick candidates within our LINER sample, black-filled stars are Compton-thin Seyferts, black-open stars are Compton-thick Seyferts, blue triangles are type 1 Seyferts (Seyferts taken from by Panessa et al. 2006), upside-down black triangles are PG QSOs reported by Jimenez-Bailon et al. (2005) and Piconcelli et al. (2005). The linear fit for LINER nuclei is shown as a dot-dashed line while the fit for all the AGN families is shown as a continuous line. The correlation coefficient ( $\mathrm{r}$ ) is given in the bottom-right text of each panel. 


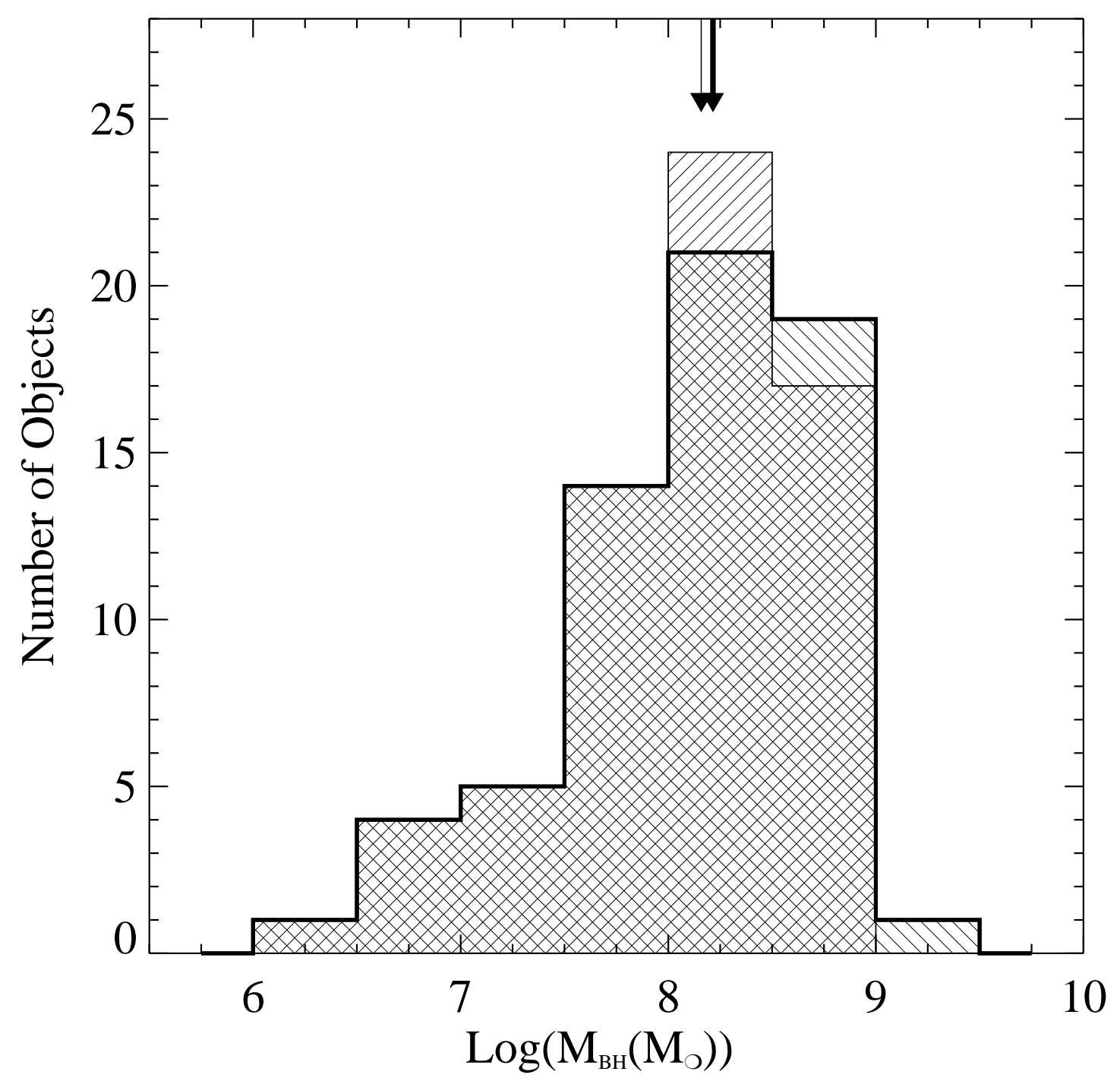

Fig. 9.- Histogram for black hole masses. The thick-filled region (top-left to bottomright lines) shows the distribution obtained using the relation by Tremaine et al. (2002) and the thin filled region (bottom-left to top-right lines) show the distribution using that by Ferrarese et al. (2001). Black thick and thin arrows show the locii of the median value using Tremaine et al. (2002) and Ferrarese et al. (2001), respectively. 


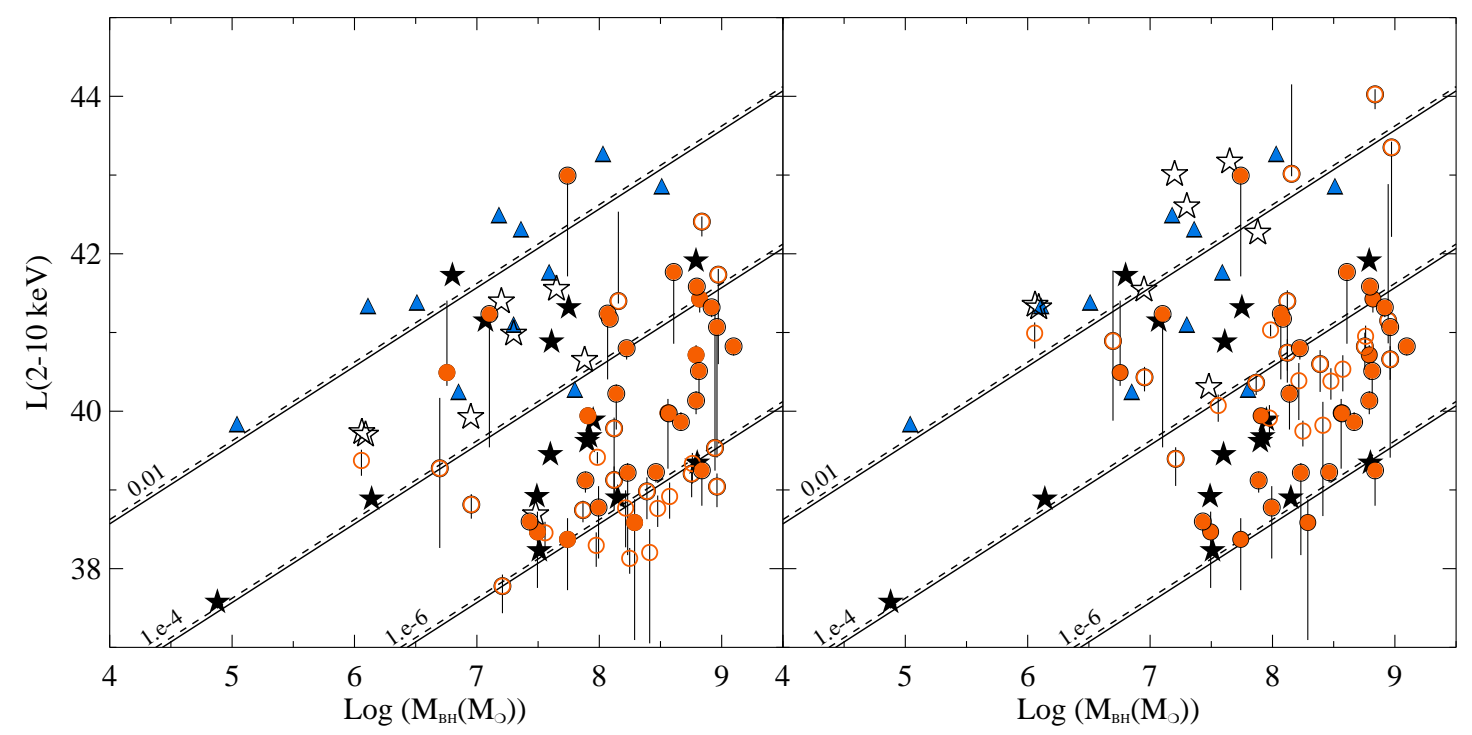

Fig. 10.- Hard X-rays (2-10 keV) luminosities versus the black hole masses, before (Left) and after (Right) Compton-thickness correction. Symbols as in Fig. 8. The solid and dashed lines show the $\mathrm{L}(2-10 \mathrm{keV})$ luminosity as a function of the black hole mass for Eddington ratios of $0.01,10^{-4}$ and $10^{-6}$ (assuming $\mathrm{L}_{\text {bol }} / \mathrm{L}(2-10 \mathrm{keV})=34$ and $\mathrm{L}_{\text {bol }} / \mathrm{L}(2-10 \mathrm{keV})=30$, Risaliti and Elvis 2004; Panessa et al. 2006, respectively). 
Table 1:: Properties of the LINER sample.

\begin{tabular}{|c|c|c|c|c|c|c|c|c|c|c|c|}
\hline $\begin{array}{l}\text { Name } \\
\text { (1) } \\
\end{array}$ & $\begin{array}{l}\text { RA } \\
(2) \\
\end{array}$ & $\begin{array}{l}\text { Dec } \\
(3) \\
\end{array}$ & $\begin{array}{l}\mathrm{N}_{\mathrm{H} 1} \\
(4) \\
\end{array}$ & $\begin{array}{l}\mathrm{N}_{\mathrm{H} 2} \\
(5) \\
\end{array}$ & $\begin{array}{c}\log L_{X} \\
(6)\end{array}$ & $\begin{array}{c}\mathrm{F}([\mathrm{OIII}]) \\
(7)\end{array}$ & $\begin{array}{l}\mathrm{Av} \\
(8) \\
\end{array}$ & $\begin{array}{r}\text { HST } \\
(9) \\
\end{array}$ & $\begin{array}{l}\mathrm{M}_{\mathrm{BH}} \\
(10) \\
\end{array}$ & $\begin{array}{c}\operatorname{Ref}(\sigma) \\
(11) \\
\end{array}$ & $\begin{array}{c}\text { Envir. } \\
(12) \\
\end{array}$ \\
\hline NGC 0315 & 005748.88 & +002108.8 & $15.21 \begin{array}{l}21.33 \\
10.67\end{array}$ & $1.06_{0.87}^{1.40}$ & 41.77 & 0.53 & $\ldots$ & $\mathrm{C}$ & 8.61 & TD81 & 20 \\
\hline NGC 0410 . & 011058.87 & +030908.3 & $0.12 \begin{array}{l}0.17 \\
0.09\end{array}$ & $0.01 \begin{array}{l}0.20 \\
0.01\end{array}$ & 40.72 & 0.35 & $\ldots$ & & 8.79 & TD81 & 20 \\
\hline NGC $0474 \ldots \ldots$ & 012006.70 & +002455.0 & $\ldots$ & $\ldots$ & $<40.26$ & 0.34 & 0.61 & & 7.93 & TD81 & 2.5 \\
\hline IIIZW 035 & 014430.50 & +170605.0 & $\ldots$ & $\ldots$ & 41.83 & 19.57 & 1.54 & $\mathrm{C}$ & & $\ldots$ & 1.5 \\
\hline NGC $0524 \ldots \ldots$ & 012447.72 & +003219.8 & $\ldots$ & $\ldots$ & 38.59 & 0.05 & $\ldots$ & $\mathrm{D}$ & 8.29 & TD81 & 5 \\
\hline NGC $0833 \ldots \ldots$ & 020920.88 & +100800.3 & $3.51_{0.73}^{10.79}$ & $26.88_{1.34}^{41.78}$ & 41.73 & 3.50 & 1.38 & & 8.97 & OFJSB & 4 \\
\hline NGC $0835 \ldots \ldots$ & 020924.69 & -100810.5 & $0.16 \begin{array}{l}3.13 \\
0.01\end{array}$ & $40.35 \begin{array}{l}1.34 .46 \\
24.60\end{array}$ & 41.40 & $\ldots$ & $\ldots$ & & 8.16 & TD81 & 4 \\
\hline NGC $1052 \ldots \ldots$ & 024104.80 & +08 1520.8 & 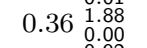 & $12.90 \begin{array}{l}35.47 \\
0.00\end{array}$ & 41.24 & 33.07 & $\ldots$ & $\mathrm{C}$ & 8.07 & CDB-93 & 5 \\
\hline NGC $2639 \ldots \ldots$ & 084338.08 & +501220.0 & $0.80 \begin{array}{l}0.92 \\
0.67\end{array}$ & $\ldots$ & $<40.06$ & 4.74 & 0.78 & $\mathrm{C}$ & 7.85 & $\mathrm{~S} 83$ & 0 \\
\hline NGC 2655 & 085537.73 & +781323.1 & $0.01 \begin{array}{c}0.07 \\
0.01\end{array}$ & $30.20 \begin{array}{l}39.47 \\
24.21\end{array}$ & 41.23 & 19.72 & 1.35 & $\mathrm{C}$ & 7.10 & OFJSB & 1.5 \\
\hline NGC $2681 \ldots \ldots$ & 085332.73 & +511849.3 & $0.15_{0.01}^{0.28} 0$ & 0.010 .08 & 41.05 & 4.94 & 1.25 & $\mathrm{C}$ & 6.70 & OFJSB & 4 \\
\hline NGC $2685 \ldots \ldots$. & 085534.75 & +584403.9 & & $\ldots$ & 40.82 & 5.28 & 1.56 & & 8.96 & $\operatorname{Din}+95$ & $0 ?$ \\
\hline UGC 4881 . & 091555.10 & +441955.0 & $0.61_{0.32}^{0.79}$ & $\ldots$ & $<40.15$ & 7.39 & 1.84 & $\mathrm{C}$ & $\ldots$ & $\ldots$ & 1.5 \\
\hline $3 \mathrm{C} 218 \ldots \ldots \ldots$ & 091805.67 & -120544.0 & $0.07 \begin{array}{l}0.20 \\
0.04\end{array}$ & $4.05 \begin{array}{l}5.96 \\
2.88\end{array}$ & 42.08 & 0.38 & $\ldots$ & & $\ldots$ & $\ldots$ & 20 \\
\hline NGC 2787 . & 091918.56 & +691212.0 & $0.11_{0.03}^{0.22}$ & & $<38.81$ & 1.17 & $\ldots$ & $\mathrm{C}$ & 8.11 & TD81 & 0 \\
\hline NGC $2841 \ldots \ldots$ & 092202.63 & +505835.5 & $0.01 \begin{array}{l}0.09 \\
0.01\end{array}$ & $3.30 \begin{array}{l}5.60 \\
1.96\end{array}$ & 39.22 & 1.41 & 0.22 & $\mathrm{C}$ & 8.23 & WRF84 & 0 \\
\hline UGC $05101 \ldots \ldots$ & 093551.65 & +612111.3 & $0.21 \begin{array}{l}0.61 \\
0.04\end{array}$ & $135.07 \begin{array}{l}380.33 \\
43.51\end{array}$ & 43.84 & 561.20 & 4.67 & $\mathrm{C}$ & & $\ldots$ & 1.5 \\
\hline NGC $3185 \ldots \ldots$ & 101738.57 & +2141 17.7 & $\ldots$ & $\ldots$ & 41.15 & 24.89 & 1.33 & $\mathrm{C}$ & 6.06 & NW95 & 4 \\
\hline NGC $3226 \ldots \ldots$ & 102327.01 & +195354.7 & $0.21{ }_{0.11}^{0.30}$ & $1.22 \begin{array}{l}1.76 \\
0.94\end{array}$ & 40.80 & 1.33 & $\ldots$ & & 8.22 & TD81 & 1.5 \\
\hline NGC $3245 \ldots \ldots$ & 102718.39 & +283026.6 & $\ldots$ & $\ldots$ & 40.76 & 3.81 & 1.24 & $\mathrm{C}$ & 8.39 & TD81 & 2.5 \\
\hline NGC $3379 \ldots \ldots$ & 104749.60 & +123453.9 & $\ldots$ & $\ldots$ & 39.91 & 0.68 & $\ldots$ & $\mathrm{C}$ & 8.25 & TD81 & 4.5 \\
\hline NGC $3414 \ldots \ldots$ & 105116.23 & +275830.0 & $0.21 \begin{array}{l}0.30 \\
0.13\end{array}$ & $\ldots$ & 39.86 & 1.85 & 0.15 & & 8.67 & $\operatorname{Din}+95$ & 5 \\
\hline NGC 3507 . & 110325.39 & +180807.4 & $0.08 \begin{array}{l}0.39 \\
0.01\end{array}$ & $\ldots$ & $<38.98$ & 2.79 & 0.60 & $\mathrm{C}$ & & $\ldots$ & 2.5 \\
\hline NGC 3607 & 111654.66 & +180306.5 & $\ldots$ & $\ldots$ & 40.54 & 5.54 & 1.69 & $\mathrm{C}$ & 8.48 & TD81 & 4.5 \\
\hline NGC 3608 & 111658.96 & +180854.9 & $\ldots$ & $\ldots$ & 39.98 & 0.28 & $\ldots$ & $\mathrm{C}$ & 8.41 & TD81 & 5 \\
\hline NGC $3623 \ldots \ldots$ & 111855.96 & +130532.0 & $\ldots$ & $\ldots$ & $<39.38$ & 0.76 & $\ldots$ & $\mathrm{C}$ & 7.62 & WKS & 3 \\
\hline NGC $3627 \ldots \ldots$ & 112015.03 & +125929.6 & $\ldots$ & $\ldots$ & 41.19 & 27.92 & 1.87 & $\mathrm{D}$ & 7.98 & WKS & 3 \\
\hline NGC $3628 \ldots \ldots$ & 112017.01 & +133522.9 & $0.46^{0.52} 0.41$ & $\ldots$ & 39.94 & 0.17 & 1.22 & $\mathrm{U}$ & 7.91 & WKS & 3 \\
\hline NGC 3690B ..... & 112832.20 & +583344.0 & $0.21 \begin{array}{c}0.41 \\
0.03\end{array}$ & $9.49 \frac{12.54}{7.52}$ & 42.64 & 205.60 & 1.17 & $\mathrm{C}$ & $\ldots$ & $\ldots$ & 1.5 \\
\hline NGC $3898 \ldots \ldots$ & 114915.37 & +560503.7 & $\begin{array}{ll}1.39 & 1.75 \\
1.12\end{array}$ & $0.01 \begin{array}{c}0.59 \\
0.01\end{array}$ & $<40.55$ & 0.90 & $\ldots$ & $\mathrm{C}$ & 8.29 & S 83 & 20 \\
\hline NGC $3945 \ldots \ldots$ & 115313.73 & +604032.0 & $0.04 \begin{array}{l}0.17 \\
0.01\end{array}$ & $\ldots .01$ & 39.12 & 0.55 & 0.27 & $\mathrm{C}$ & 7.89 & OFJSB & 0 \\
\hline NGC $3998 \ldots \ldots$. & 115756.12 & +552712.7 & $0.08 \begin{array}{l}0.15 \\
0.06\end{array}$ & $2.30 \begin{array}{l}3.18 \\
1.63\end{array}$ & 41.32 & 32.16 & 1.22 & $\mathrm{C}$ & 8.92 & TD81 & 5 \\
\hline NGC $4036 \ldots \ldots$ & 120126.75 & +615344.8 & & $\ldots$ & 40.90 & 2.01 & $\ldots$ & $\mathrm{C}$ & 8.12 & TD81 & 2.5 \\
\hline NGC $4111 \ldots \ldots$ & 120703.13 & +430355.4 & $4.67 \begin{array}{l}9.65 \\
1.20\end{array}$ & $37.71_{12.52}^{100.00}$ & $<40.36$ & 5.87 & 1.25 & $\mathrm{C}$ & 7.56 & OFJSB & 5 \\
\hline NGC $4125 \ldots \ldots$ & 120806.02 & +651026.9 & $0.53 \begin{array}{c}0.88 \\
0.01\end{array}$ & $0.86 \begin{array}{l}2.13 \\
0.08\end{array}$ & $<40.51$ & 0.73 & $\ldots$ & $\mathrm{C}$ & 8.31 & TD81 & 2 \\
\hline IRAS $12112+0305$ & 121346.00 & +024838.0 & & $\ldots$ & 43.00 & 2.36 & 1.11 & & $\ldots$ & $\ldots$ & 1 \\
\hline NGC $4261 \ldots \ldots$ & 121923.22 & +054930.8 & $0.69 \begin{array}{l}1.38 \\
0.31\end{array}$ & $16.45_{13.25}^{21.64}$ & 41.07 & 3.71 & 1.33 & $\mathrm{U}$ & 8.96 & TD81 & 5 \\
\hline NGC $4278 \ldots \ldots$ & 122006.83 & +291650.7 & $0.09 \begin{array}{l}0.12 \\
0.06\end{array}$ & $2.65 \begin{array}{l}1.32 \\
1.48\end{array}$ & 41.00 & 6.72 & $\ldots$ & $\mathrm{C}$ & 8.46 & TD81 & 5 \\
\hline NGC $4314 \ldots \ldots$ & 122231.99 & +295343.3 & $0.27 \begin{array}{l}0.44 \\
0.09\end{array}$ & $0.01 \begin{array}{l}0.52 \\
0.01\end{array}$ & $<39.10$ & 0.74 & 0.32 & $\mathrm{C}$ & 7.19 & BHS02r & 5 \\
\hline
\end{tabular}


Table 1:: Continuation

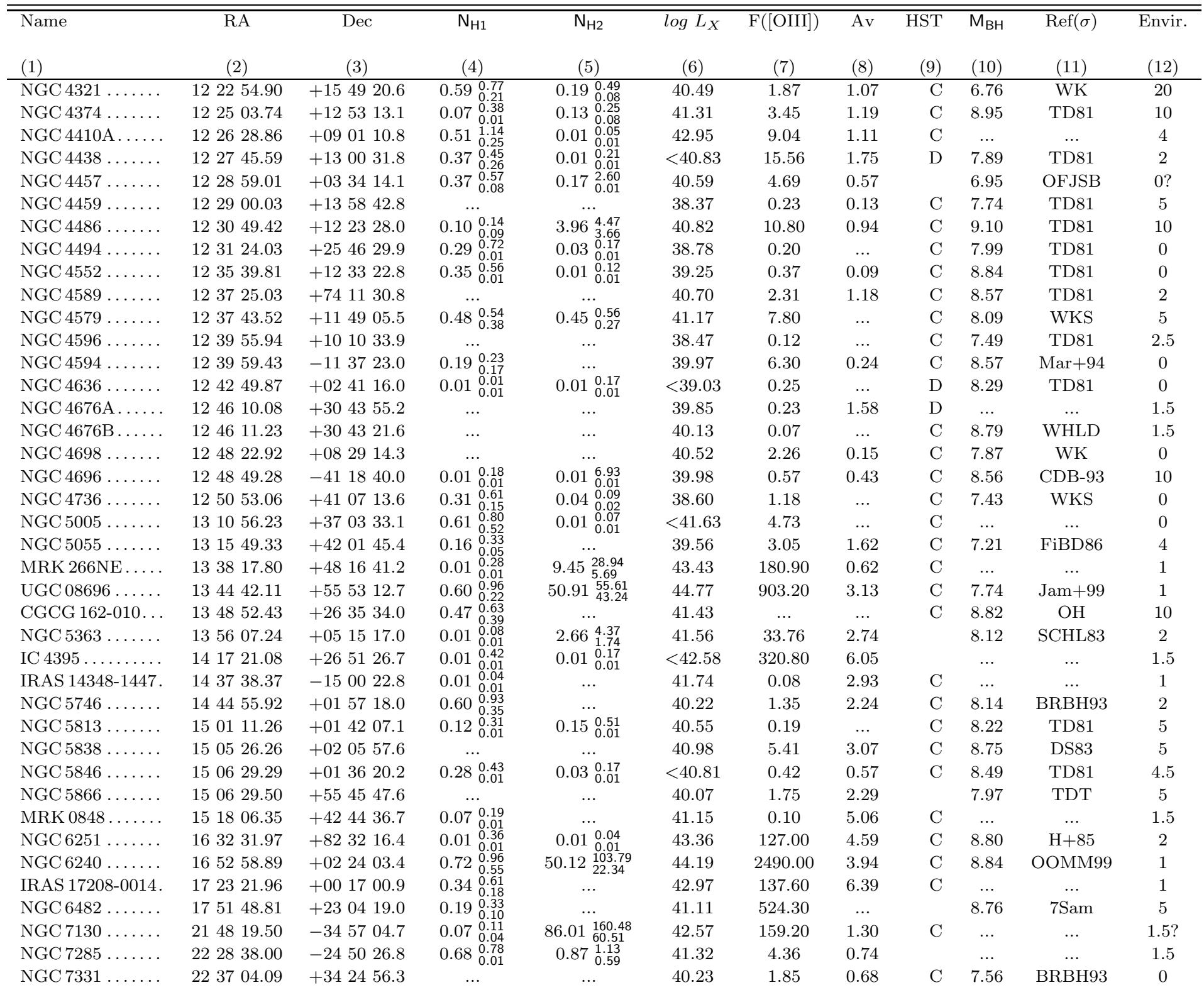


Table 1:: Continuation

\begin{tabular}{|c|c|c|c|c|c|c|c|c|c|c|c|}
\hline Name & $\overline{\mathrm{RA}}$ & $\overline{\overline{\text { Dec }}}$ & $\overline{\mathrm{N}_{\mathrm{H} 1}}$ & $\overline{\mathrm{N}_{\mathrm{H} 2}}$ & $\overline{l \log L_{X}}$ & $\overline{\mathrm{F}([\mathrm{OIII}])}$ & $\overline{\mathrm{Av}}$ & 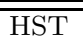 & $\overline{\mathrm{M}_{\mathrm{BH}}}$ & $\overline{\operatorname{Ref}(\sigma)}$ & Envir. \\
\hline (1) & (2) & (3) & (4) & (5) & (6) & (7) & (8) & (9) & $(10)$ & (11) & (12) \\
\hline IC 1459. & $2257 \quad 10.60$ & -362744.0 & $0.20_{0.09}^{0.28}$ & $1.26_{0.66}^{3.17}$ & 40.51 & . & & $\mathrm{C}$ & 8.81 & CDB-93 & 4 \\
\hline NPM1G-12.0625 & 232519.82 & -120726.4 & $0.71 \begin{array}{l}0.85 \\
0.33\end{array}$ & $0.15^{0.32} 0$ & $<43.24$ & 2.42 & 1.06 & $\mathrm{D}$ & 8.18 & SHI90 & 10 \\
\hline NGC $7743 \ldots \ldots$ & 234421.14 & +095602.7 & $0.35 \begin{array}{l}0.58 \\
0.18\end{array}$ & 1.683 .63 & $<41.33$ & 46.37 & 1.84 & $\mathrm{C}$ & 6.62 & Kor82 & 0 \\
\hline
\end{tabular}

Name (Col. 1), position (2000) (Cols. 2 and 3), soft (NH1, Col. 5) and hard (NH2, Col. 6) column densities in units of 1022 $\mathrm{cm}^{-2}$, logarith of the hard (2-10 keV) Xray luminosity (Col. 7), [OIII] emission line flux corrected for reddening in units of $1 \times 10^{-14} \mathrm{erg} \mathrm{s}^{-1} \mathrm{~cm}^{-2}$.(Col. 8), optical extinction Av (Col. 9), HST morphology (Col. 10), black hole mass in logarithmical scale and their reference (Cols. 11 and 12) and environmental classification $(\mathrm{Col} . \quad 13) . \mathrm{Av}=6.67 \mathrm{log}(\mathrm{H} \alpha / \mathrm{Rv} * \mathrm{H} \beta)$. F([OIII]) measured by Ho et al. (2001); Moustakas and Kennicutt (2006); Veilleux et al. (1995); Keel et al. (1985); Keel (1983); Koski (1978); Greenawalt et al. (1997); Duc et al. (1997). Column (12) Interacting types: $0=$ Isolate, $1=$ Merger, $1.5=$ Close Interacting Pair, $2=$ Pair, $2.5=$ Wide Pair, $3=$ Triplet, $4=$ Compact Group, $4.5=1$ st group, $5=$ Group, $10=$ Cluster Center and, $20=$ Cluster Member 
Table 2:: Summary of Compton-thick signatures.

\begin{tabular}{|c|c|c|c|c|c|c|c|c|c|}
\hline \multirow{2}{*}{$\begin{array}{l}\text { Name } \\
(1) \\
\end{array}$} & \multirow{2}{*}{$\begin{array}{l}\Gamma \\
(2)\end{array}$} & \multirow{2}{*}{$\begin{array}{c}C-T 1^{*} \\
(3)\end{array}$} & \multirow{2}{*}{$\begin{array}{c}\mathrm{F}_{\mathrm{X}} / \mathrm{F}([\mathrm{OIII}]) \\
(4)\end{array}$} & \multirow{2}{*}{$\begin{array}{c}C-\text { T2* }^{*} \\
(5)\end{array}$} & \multicolumn{3}{|c|}{$\overline{\mathrm{EW}(\mathrm{FeK} \alpha)}$} & \multirow{2}{*}{$\begin{array}{c}\text {-T3* } \\
(9)\end{array}$} & \multirow{2}{*}{$\begin{array}{l}C-T \\
(10)\end{array}$} \\
\hline & & & & & $\begin{array}{c}\text { Best-fit } \\
(6)\end{array}$ & $\begin{array}{c}\text { Pexrav } \\
(7)\end{array}$ & $\begin{array}{c}\text { Baseline CT } \\
(8)\end{array}$ & & \\
\hline NGC $0315 \ldots . .$. & $1.58_{1.42}^{2.08}$ & & 2.30 & & $80_{1}^{160}$ & $<100$ & $<150$ & & \\
\hline NGC $0410 \ldots \ldots$ & $>2.86$ & & 1.38 & & $<50$ & $<580$ & $<480$ & & \\
\hline NGC $0474 \ldots \ldots$ & $\ldots$ & $?$ & -0.13 & $\mathrm{CT}$ & $\ldots$ & $\ldots$ & $\ldots$ & $?$ & $\mathrm{CT}$ \\
\hline IIIZW $035 \ldots \ldots$ & $\ldots$ & $?$ & -1.34 & $\mathrm{CT}$ & $\ldots$ & $\ldots$ & $\ldots$ & $?$ & $\mathrm{CT}$ \\
\hline NGC $0524 \ldots \ldots$ & $\ldots$ & $?$ & 1.09 & & $\ldots$ & $\ldots$ & $\ldots$ & $?$ & \\
\hline NGC $0833 \ldots \ldots$ & $\cdots$ & $?$ & 1.78 & & $330{ }_{50}^{620}$ & $\cdots$ & $<370$ & $\mathrm{CT}$ & CT? \\
\hline NGC $0835 \ldots \ldots$ & $\ldots$ & $?$ & $\ldots$ & $?$ & $770 \begin{array}{l}50 \\
480\end{array}$ & $630 \begin{array}{l}880 \\
380\end{array}$ & $610 \begin{array}{l}850 \\
360\end{array}$ & $\mathrm{CT}$ & $\mathrm{CT}$ \\
\hline NGC $1052 \ldots \ldots$ & $1.27 \begin{array}{l}1.32 \\
1.18\end{array}$ & $\mathrm{CT}$ & 1.07 & & $140 \begin{array}{l}170 \\
120\end{array}$ & 7090 & $110 \begin{array}{l}130 \\
90\end{array}$ & & \\
\hline NGC $2639 \ldots \ldots$ & $\ldots$ & $?$ & -1.76 & $\mathrm{CT}$ & $\ldots$ & $\ldots$ & $\ldots$ & $?$ & $\mathrm{CT}$ \\
\hline NGC $2655 \ldots \ldots$. & $2.24 \begin{array}{l}4.26 \\
1.88\end{array}$ & & 1.14 & & $<160$ & $<140$ & $<110$ & & \\
\hline NGC $2681 \ldots \ldots$ & $\begin{array}{l}1.514 .58 \\
0.66\end{array}$ & $\mathrm{CT}$ & 0.08 & CT? & $<2210$ & $\ldots$ & $<1430$ & $\mathrm{CT}$ & $\mathrm{CT}$ \\
\hline NGC $2685 \ldots \ldots$. & $\ldots$ & $?$ & -0.11 & $\mathrm{CT}$ & $\ldots$ & $\ldots$ & $\ldots$ & $?$ & $\mathrm{CT}$ \\
\hline UGC $4881 \ldots \ldots$ & $\ldots$ & $?$ & -2.89 & $\mathrm{CT}$ & $\ldots$ & $\ldots$ & $\ldots$ & $?$ & $\mathrm{CT}$ \\
\hline $3 \mathrm{C} 218 \ldots \ldots \ldots$ & $2.60 \begin{array}{l}2.79 \\
2.50\end{array}$ & & 1.75 & & $<10$ & $<3$ & $<10$ & & \\
\hline NGC $2787 \ldots \ldots$ & $\begin{array}{l}3.27 \begin{array}{r}5.16 \\
2.02\end{array} \\
3\end{array}$ & & 0.91 & & $<290$ & $<250$ & $<190$ & & \\
\hline NGC $2841 \ldots \ldots$ & $1.95 \begin{array}{l}3.64 \\
1.26\end{array}$ & & 0.84 & & $<240$ & $<280$ & $<400$ & & \\
\hline UGC $05101 \ldots \ldots$ & $0.30_{-0.36}^{0.98}$ & $\mathrm{CT}$ & -0.96 & $\mathrm{CT}$ & $280 \begin{array}{l}460 \\
100\end{array}$ & $320 \begin{array}{l}500 \\
130\end{array}$ & $320{ }_{140}^{510}$ & & $\mathrm{CT}$ \\
\hline NGC $3185 \ldots \ldots$. & $\ldots$ & $?$ & -0.70 & $\mathrm{CT}$ & $\ldots$ & $\ldots$ & $\ldots$ & $?$ & $\mathrm{CT}$ \\
\hline NGC $3226 \ldots \ldots$ & $1.81_{1.61}^{2.24}$ & & 1.85 & & $<110$ & $<90$ & $<100$ & & \\
\hline NGC $3245 \ldots \ldots$. & $\ldots$ & $?$ & -0.26 & $\mathrm{CT}$ & $\ldots$ & $\ldots$ & $\ldots$ & $?$ & $\mathrm{CT}$ \\
\hline NGC $3379 \ldots \ldots$ & $\ldots$ & $?$ & 0.17 & $\mathrm{CT}$ & $\ldots$ & $\ldots$ & $\ldots$ & $?$ & $\mathrm{CT}$ \\
\hline NGC $3414 \ldots \ldots$. & $>2.51$ & & 0.72 & & $<590$ & $<7790$ & $<750$ & $\mathrm{CT}$ & \\
\hline NGC $3507 \ldots \ldots$ & $\ldots$ & $?$ & -1.89 & $\mathrm{CT}$ & $\ldots$ & $\ldots$ & $\ldots$ & $?$ & $\mathrm{CT}$ \\
\hline NGC $3607 \ldots \ldots$ & $\ldots$ & $?$ & -0.70 & $\mathrm{CT}$ & $\ldots$ & $\ldots$ & $\ldots$ & $?$ & $\mathrm{CT}$ \\
\hline NGC $3608 \ldots . .$. & $\ldots$ & $?$ & -0.04 & $\mathrm{CT}$ & $\ldots$ & $\ldots$ & $\ldots$ & $?$ & $\mathrm{CT}$ \\
\hline NGC $3623 \ldots \ldots$ & $\ldots$ & $?$ & 1.70 & & $\ldots$ & $\ldots$ & $\ldots$ & $?$ & \\
\hline NGC $3627 \ldots \ldots$ & $\ldots$ & $?$ & -0.05 & $\mathrm{CT}$ & $\ldots$ & $\ldots$ & $\ldots$ & $?$ & $\mathrm{CT}$ \\
\hline NGC $3628 \ldots \ldots$ & $1.56_{1.38}^{2.10}$ & & 2.92 & & $<80$ & $<80$ & $<90$ & & \\
\hline NGC $3690 \mathrm{~B} \ldots . .$. & $\ldots$ & $?$ & -0.72 & $\mathrm{CT}$ & $230 \begin{array}{l}340 \\
130\end{array}$ & $220 \begin{array}{l}330 \\
110\end{array}$ & $250 \begin{array}{l}360 \\
140\end{array}$ & & CT? \\
\hline NGC $3898 \ldots \ldots$ & $\ldots$ & $?$ & 0.06 & CT? & $\ldots$ & $\ldots$ & $\ldots$ & $?$ & $\mathrm{CT}$ \\
\hline NGC $3945 \ldots \ldots$. & $\ldots$ & $?$ & 0.61 & & $<110$ & $\ldots$ & $<2$ & & \\
\hline NGC $3998 \ldots . .$. & $1.88_{1}^{2.09}$ & & 1.48 & & $<30$ & $<20$ & $<40$ & & \\
\hline NGC $4036 \ldots .$. & $2.14 \begin{array}{l}6.54 \\
-0.97\end{array}$ & $\mathrm{CT}$ & -0.03 & $\mathrm{CT}$ & $\ldots$ & $\ldots$ & $\ldots$ & $?$ & $\mathrm{CT}$ \\
\hline NGC $4111 \ldots \ldots$ & $1.37_{0.05}^{2.31}$ & $\mathrm{CT}$ & 1.22 & & $<180$ & $<420$ & $<180$ & & \\
\hline NGC $4125 \ldots \ldots$ & $>1.58$ & & 0.35 & CT? & $<1560$ & $<780$ & $<2380$ & $\mathrm{CT}$ & $\mathrm{CT}$ \\
\hline IRAS $12112+0305$ & $\ldots$ & $?$ & -0.11 & CT? & $\ldots$ & $\ldots$ & $\ldots$ & $?$ & $\mathrm{CT}$ \\
\hline NGC $4261 \ldots \ldots$ & $1.89 \begin{array}{l}2.40 \\
1.54\end{array}$ & & 1.48 & & $<30$ & $<70$ & $<80$ & & \\
\hline NGC $4278 \ldots \ldots$ & $2.09 \begin{array}{l}1.40 \\
1.93\end{array}$ & & -0.09 & $\mathrm{CT}$ & $<50$ & $<30$ & $<50$ & & \\
\hline NGC $4314 \ldots \ldots$ & $2.52 \begin{array}{l}4.66 \\
0.06\end{array}$ & $\mathrm{CT}$ & 1.19 & & $<710$ & $\ldots$ & $<1730$ & $\mathrm{CT}$ & $\mathrm{CT}$ \\
\hline NGC 4321 & $3.22 \begin{array}{l}6.25 \\
1.64\end{array}$ & & 1.77 & & $<170$ & $<240$ & $<240$ & & \\
\hline NGC $4374 \ldots \ldots$ & $2.59 \begin{array}{l}1.04 \\
1.12\end{array}$ & $\mathrm{CT}$ & 0.44 & $\mathrm{CT} ?$ & $<1600$ & $<50$ & $<1430$ & $\mathrm{CT}$ & $\mathrm{CT}$ \\
\hline NGC $4410 \mathrm{~A} \ldots . .$. & $1.46 \begin{array}{l}3.32 \\
0.77\end{array}$ & $\mathrm{CT}$ & 0.19 & $\mathrm{CT} ?$ & $<430$ & $<300$ & $<205$ & & $\mathrm{CT}$ \\
\hline NGC $4438 \ldots \ldots$ & $9.98 \begin{array}{l}0.95 \\
0.86\end{array}$ & $\mathrm{CT}$ & -0.60 & $\mathrm{CT}$ & $<2460$ & $\ldots$ & $<2020$ & $\mathrm{CT}$ & $\mathrm{CT}$ \\
\hline NGC $4457 \ldots \ldots$ & $>0.79$ & $\mathrm{CT}$ & -0.39 & $\mathrm{CT}$ & $<60$ & $\ldots$ & $<60$ & & $\mathrm{CT}$ \\
\hline NGC $4459 \ldots \ldots$ & $\ldots$ & $?$ & 0.52 & & $\ldots$ & $\ldots$ & $\ldots$ & $?$ & \\
\hline NGC $4486 \ldots .$. & $2.79_{2.77}^{2.81}$ & & 1.34 & & $90 \stackrel{130}{40}$ & $<50$ & $<50 \frac{100}{10}$ & & \\
\hline NGC $4494 \ldots \ldots$ & $1.91 \begin{array}{l}2.94 \\
1.34\end{array}$ & & 0.93 & & $<370$ & $\ldots$ & $<220$ & & \\
\hline NGC $4552 \ldots \ldots$ & $2.11 \begin{array}{l}3.58 \\
1.82\end{array}$ & & 1.23 & & $<320$ & $<420$ & $<390$ & & \\
\hline NGC 4589 & $\ldots$ & $?$ & -0.16 & $\mathrm{CT}$ & $\ldots$ & $\ldots$ & $\ldots$ & $?$ & $\mathrm{CT}$ \\
\hline NGC $4579 \ldots \ldots$ & $1.71 \begin{array}{l}1.80 \\
1.64\end{array}$ & & 1.75 & & $110_{70}^{160}$ & $80 \stackrel{120}{40}$ & $120{ }_{80}^{160}$ & & \\
\hline NGC $4596 \ldots \ldots$ & $\ldots$ & $?$ & 0.84 & & $\ldots$ & $\ldots$ & $\ldots$ & $?$ & \\
\hline NGC $4594 \ldots \ldots$ & $2.08 \begin{array}{l}2.36 \\
1.76\end{array}$ & & 1.13 & & $<110$ & $<80$ & $<100$ & & \\
\hline
\end{tabular}


Table 2:: Continuation

\begin{tabular}{|c|c|c|c|c|c|c|c|c|c|}
\hline \multirow{2}{*}{$\begin{array}{l}\text { Name } \\
(1)\end{array}$} & \multirow{2}{*}{$\begin{array}{l}\Gamma \\
(2)\end{array}$} & \multirow{2}{*}{$\begin{array}{c}C-T 1^{*} \\
(3)\end{array}$} & \multirow{2}{*}{$\begin{array}{c}\mathrm{F}_{\mathrm{X}} / \mathrm{F}([\mathrm{OIII}]) \\
(4)\end{array}$} & \multirow{2}{*}{$\begin{array}{c}C-\text { T2* }^{*} \\
(5)\end{array}$} & \multicolumn{3}{|c|}{$\overline{\mathrm{EWW}(\mathrm{FeK} \alpha)}$} & \multirow{2}{*}{$\begin{array}{c}C-T 3^{*} \\
(9)\end{array}$} & \multirow{2}{*}{$\begin{array}{l}C-T \\
(10)\end{array}$} \\
\hline & & & & & $\begin{array}{c}\text { Best-fit } \\
(6)\end{array}$ & $\begin{array}{c}\text { Pexrav } \\
(7)\end{array}$ & $\begin{array}{c}\text { Baseline CT } \\
(8)\end{array}$ & & \\
\hline NGC $4636 \ldots . .$. & $1.63_{-0.54}^{4.91}$ & $\mathrm{CT}$ & 1.21 & & $<1750$ & $<2730$ & $<1890$ & $\mathrm{CT}$ & $\mathrm{CT}$ \\
\hline NGC $4676 \mathrm{~A} \ldots . .$. & $\ldots$ & $?$ & 0.58 & & $\ldots$ & $\ldots$ & $\ldots$ & $?$ & \\
\hline NGC 4676B. & $\ldots$ & $?$ & 1.33 & & $\ldots$ & $\ldots$ & $\ldots$ & $?$ & \\
\hline NGC $4698 \ldots \ldots$ & $\ldots$ & $?$ & -0.13 & $\mathrm{CT}$ & $\ldots$ & $\ldots$ & $\ldots$ & $?$ & $\mathrm{CT}$ \\
\hline NGC $4696 \ldots \ldots$ & $3.65 \begin{array}{l}3.75 \\
3.54\end{array}$ & & 1.06 & & $\ldots$ & $\ldots$ & $\ldots$ & $?$ & \\
\hline NGC $4736 \ldots \ldots$ & 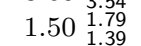 & & 1.02 & & $<100$ & $<40$ & $<50$ & & \\
\hline NGC $5005 \ldots \ldots$ & $2.03 \begin{array}{l}3.63 \\
1.30\end{array}$ & & 0.45 & CT? & $\ldots$ & $\ldots$ & $\ldots$ & $?$ & CT? \\
\hline NGC $5055 \ldots \ldots$ & $>-0.38$ & $\mathrm{CT}$ & -0.42 & $\mathrm{CT}$ & $<40$ & $\ldots$ & $<1$ & & $\mathrm{CT}$ \\
\hline MRK 266 NE ..... & & $?$ & -0.75 & $\mathrm{CT}$ & $280 \begin{array}{l}480 \\
70\end{array}$ & $210_{20}^{390}$ & $240 \quad 40$ & & CT? \\
\hline UGC $08696 \ldots \ldots$ & $0.33_{-0.42}^{0.85}$ & $\mathrm{CT}$ & 2.19 & & $270 \begin{array}{l}380 \\
150\end{array}$ & $240 \begin{array}{l}350 \\
120\end{array}$ & $250 \begin{array}{l}360 \\
130\end{array}$ & & \\
\hline CGCG $162-010 \ldots$ & $2.54 \begin{array}{l}2.60 \\
2.45\end{array}$ & & $\ldots$ & $?$ & $<10$ & $<1$ & $<5$ & & \\
\hline NGC 5363 & 1.512 .43 & $\mathrm{CT}$ & -0.41 & $\mathrm{CT}$ & $<340$ & $<280$ & $<230$ & & $\mathrm{CT}$ \\
\hline IC $4395 \ldots \ldots \ldots$ & $>1.13$ & $\mathrm{CT}$ & -1.88 & $\mathrm{CT}$ & $\ldots$ & $\ldots$ & $\ldots$ & $?$ & $\mathrm{CT}$ \\
\hline IRAS 14348-1447. & $>1.33$ & & 1.73 & & $\ldots$ & $\ldots$ & $\ldots$ & $?$ & \\
\hline NGC $5746 \ldots \ldots$ & $1.51 \begin{array}{l}2.68 \\
1.12\end{array}$ & $\mathrm{CT}$ & 1.17 & & $<380$ & $<370$ & $<400$ & & \\
\hline NGC $5813 \ldots \ldots$ & $>4.14$ & & 0.40 & CT? & $<2610$ & $<8680$ & $<4880$ & $\mathrm{CT}$ & $\mathrm{CT}$ \\
\hline NGC $5838 \ldots \ldots$ & $\ldots$ & $?$ & -0.38 & $\mathrm{CT}$ & $\ldots$ & $\ldots$ & $\ldots$ & $?$ & $\mathrm{CT}$ \\
\hline NGC $5846 \ldots \ldots$ & $2.32 \begin{array}{l}3.18 \\
1.71\end{array}$ & & 2.35 & & $<180$ & $\ldots$ & $<200$ & & \\
\hline NGC $5866 \ldots \ldots$ & $\ldots$ & $?$ & -0.30 & $\mathrm{CT}$ & $\ldots$ & $\ldots$ & $\ldots$ & $?$ & $\mathrm{CT}$ \\
\hline MRK $0848 \ldots . .$. & $1.47 \begin{array}{l}4.40 \\
0.00\end{array}$ & $\mathrm{CT}$ & 1.66 & & $<2400$ & $<1020$ & $<30840$ & $\mathrm{CT}$ & $\mathrm{CT}$ \\
\hline NGC $6251 \ldots \ldots$ & 1.821 .89 & & -0.33 & $\mathrm{CT}$ & $<50$ & $<20$ & $<50$ & & \\
\hline NGC $6240 \ldots \ldots$ & $0.64 \begin{array}{l}0.93 \\
0.52\end{array}$ & CT & -0.88 & $\mathrm{CT}$ & $380 \begin{array}{l}440 \\
310\end{array}$ & $410 \begin{array}{l}480 \\
350\end{array}$ & $460{ }_{390}^{530}$ & & $\mathrm{CT}$ \\
\hline IRAS $17208-0014$ & $1.49_{-1.79}^{6.52}$ & $\mathrm{CT}$ & -1.23 & $\mathrm{CT}$ & $<560$ & $\begin{array}{l}<6320 \\
\end{array}$ & $<1330$ & $\mathrm{CT}$ & $\mathrm{CT}$ \\
\hline NGC 6482 & & $?$ & -2.90 & $\mathrm{CT}$ & & $\ldots$ & $\ldots$ & $?$ & $\mathrm{CT}$ \\
\hline NGC 7130 & $0.12_{-0.86}^{0.49}$ & $\mathrm{CT}$ & -1.05 & $\mathrm{CT}$ & $380 \begin{array}{l}630 \\
130\end{array}$ & $480 \begin{array}{l}760 \\
200\end{array}$ & $420 \begin{array}{l}690 \\
160\end{array}$ & $\mathrm{CT}$ & $\mathrm{CT}$ \\
\hline NGC 7285 & $1.82 \begin{array}{l}2.88 \\
1.40\end{array}$ & & 1.12 & & 210380 & $<300$ & 170320 & & \\
\hline NGC $7331 \ldots \ldots$ & $\ldots$ & $?$ & -0.10 & $\mathrm{CT}$ & $\ldots$ & $\ldots$ & $\ldots$ & $?$ & $\mathrm{CT}$ \\
\hline IC $1459 \ldots \ldots \ldots$ & $2.63 \begin{array}{l}2.94 \\
2.04\end{array}$ & & $\ldots$ & $?$ & $<70$ & $<80$ & $<120$ & & \\
\hline NPM1G-12.0625 & $3.00 \begin{array}{r}3.04 \\
2.89\end{array}$ & & 0.01 & CT? & $<4$ & $<2$ & $<3$ & & \\
\hline NGC $7743 \ldots \ldots$ & $>-3.00$ & $\mathrm{CT}$ & -0.75 & $\mathrm{CT}$ & $<8340$ & $\ldots$ & $<8240$ & $\mathrm{CT}$ & $\mathrm{CT}$ \\
\hline
\end{tabular}

Spectral Index (Col. 2), $\mathrm{F}_{\mathrm{X}}(2-10 \mathrm{keV}) / \mathrm{F}([\mathrm{OIII}])$ (Col. 4), $\mathrm{EW}(\mathrm{FeK} \alpha)$ for best-fit, pexrav model above $2 \mathrm{keV}$ and the baseline model of Compton-thick AGN by Guainazzi et al. (2005) (Cols. 6, 7 and 8, respectively). C-T1 = Compton-Thick candidates throught flat spectrum. $C$ - T2 $=$ Compton-Thick candidates throught $\mathrm{F}_{\mathrm{X}}(2-10 \mathrm{keV}) / \mathrm{F}([\mathrm{OIII}])$ ratio. C-T3= Compton-Thick candidates throught EW(FeKa). $C-T=$ Final Compton-Thick candidates. \&: NGC 0833 is a Compton-thin object with the baseline model of Compton-thick AGN. \$: UGC 05101 and NGC 6240 are Compton-thick objects with the baseline model of Compton-thick AGN.

Table 3: $F_{\mathbf{X}}(2-10 \mathrm{keV}) / \mathrm{F}([\mathrm{OIII}])$ ratios according to different environments.

\begin{tabular}{lccr}
\hline \hline & & & \\
Environment $(\mathrm{Nr})$ & $\mathrm{F}_{\mathbf{X}}(2-10 \mathrm{keV}) / \mathrm{F}([\mathrm{OIII}])$ & $\sigma$ & range \\
\hline ISO (15) & 0.33 & 0.77 & -1.76 to 1.23 \\
PAIR (29) & -0.06 & 1.02 & -2.89 to 2.19 \\
GROUP(23) & 0.43 & 0.91 & -2.90 to 2.35 \\
CLUSTER (9) & 1.12 & 1.12 & 0.01 to 2.30 \\
\hline
\end{tabular}

